\title{
MODELIZACIÓN ESPACIALMENTE DISTRIBUIDA DE LA EROSIÓN Y EL TRANSPORTE DE SEDIMENTO EN CUENCAS DE MONTAÑA DEL PIRINEO ARAGONÉS: RETOS PARA LA CALIBRACIÓN Y VALIDACIÓN
}

\author{
L.C. ALATORRE ${ }^{* *}$, S. BEGUERÍA ${ }^{2}$, \\ N. LANA-RENAULT ${ }^{3}$, A. NAVAS ${ }^{2}$ \\ Geoinformática, División Multidisciplinaria de la UACJ en Cuauhtémoc, \\ Universidad Autónoma de Ciudad Juárez, 31579 Chihuahua, México. \\ ${ }^{2}$ Estación Experimental de Aula Dei, Consejo Superior de Investigaciones Científicas (CSIC), \\ 50059 Zaragoza, España. \\ ${ }^{3}$ Área de Geografía, Departamento de Ciencias Humanas, Universidad de La Rioja, \\ 26004 Logroño, España.
}

RESUMEN. Una gran parte de los modelos de erosión aplicados a escala de cuenca se basan en una representación espacialmente agregada, es decir en la subdivisión del espacio en superficies a las que se supone un comportamiento homogéneo (subcuencas, unidades hidrológicas, etc.). Esta aproximación conlleva una deseable simplificación de los cálculos, pero presenta dificultades para abordar problemas relacionados con el transporte de sedimentos y la identificación de áreas fuente de sedimento, que deben tratarse mediante una representación espacial continua. Los modelos espacialmente distribuidos, en cambio, permiten una aproximación más precisa a este problema, a costa de una mayor complejidad de cálculo. El objetivo de este trabajo es la calibración y validación del modelo WATEM/SEDEM, un modelo empírico-conceptual espacialmente distribuido, para predecir erosión y la producción de sedimentos en dos cuencas del Pirineo aragonés: i) la cuenca del embalse de Barasona (1504 km²), drenada por los ríos Ésera e Isábena, donde la base de datos para el proceso de calibración fue el registro de 3 años de sedimentos en suspensión (mayo 2005-mayo 2008) a la salida de la subcuenca del Río Isábena y para la validación se utilizó el registro histórico de sedimentación del embalse de Barasona; y, ii) la cuenca experimental de Arnás $\left(2.84 \mathrm{~km}^{2}\right)$, donde se contó para la calibración con datos espacialmente distribuidos de tasas de erosión y sedimentación derivadas de inventarios de $\mathrm{Cs}^{137}$, más el registro de seis años de sedimento en suspensión a la salida de la cuenca de Arnás para el proceso de validación. El proceso de calibración para el embalse de Barasona mostró el problema que se tiene al intentar calibrar los parámetros de capacidad de transporte con una sola variable (la exportación de sedimento a la salida de la cuenca), haciendo imposible encontrar un solo conjunto de parámetros que optimicen la función de error y 
obligando a adoptar una solución de compromiso. En la cuenca de Arnás el uso de datos espacialmente distribuidos de producción de sedimentos derivados de inventarios de $\mathrm{Cs}^{137}$ permitió calibrar los parámetros empíricos de capacidad de transporte de una forma satisfactoria, encontrando una sola combinación de valores que optimiza la función de error. Con los resultados obtenidos se demuestra que la calibración de los parámetros de capacidad de transporte es un aspecto fundamental de WATEM/SEDEM y de otros modelos similares. Para obtener una estimación fiable de la distribución espacial de los procesos de erosión y transporte de sedimento se requiere de una calibración y validación con datos espacialmente distribuidos de pérdida o ganancia de suelo que permita a su vez realizar una calibración espacialmente distribuida de los parámetros relativos a la capacidad de transporte.

\section{Spatial distributed modelling of erosion and sediment transport in mountain catchments in the Pyrenees: challenges for calibration and validation}

ABSTRACT. Most erosion models applied at basin scale are based on spatially aggregated representation, for example, in a space subdivision of the surfaces to which behavior is assumed homogeneous (sub-basins, hydrological units, etc.). This approach leads to a desirable simplification of the calculations, but presents difficulties in addressing problems related to sediment transport and identification of sediment source areas, which should be treated by a continuous spatial representation. Spatially distributed models, however, allow a more accurate approach to this problem, at the cost of an increased computational complexity. The objective of this work is the calibration and validation of the model WATEM/SEDEM, an empirical-conceptual spatially distributed model, to predict erosion and sediment yield in two watersheds in the Central Spanish Pyrenees: i) the watershed of the Barasona Reservoir $\left(1504 \mathrm{~km}^{2}\right)$, which is drained by the Ésera and Isábena Rivers, the model calibration and validation was based on the depositional history of the Barasona Reservoir and suspended sediment records over 3 years (May 2005-May 2008) at the outlet of the Isábena River; ii) the experimental Arnás catchment $\left(2.84 \mathrm{~km}^{2}\right)$, the model calibration was performed based on a dataset of soil redistribution rates derived from point ${ }^{137} \mathrm{Cs}$ inventories, allowing capture differences per land use in the main model parameters. The validation process was carried with the registration of six years of suspended sediment at the outlet of the Arnás catchment. The calibration process for watershed of the Barasona Reservoir showed the problem you have when trying to calibrate the parameters of transport capacity with a single variable (the export of sediment to the basin outlet), making impossible to find a single set of parameters that optimize the error function, making it necessary to adopt a compromise solution. For the experimental Arnás catchment the model calibration processes using spatially distributed sediment yield derived from ${ }^{137} \mathrm{Cs}$ inventories allowed calibrating the empirical parameters of transport capacity in a satisfactory way, finding a single combination of values that optimizes the error function. These results show that the calibration parameters of transport capacity are a 
fundamental aspect of the model WATEM/SEDEM and other similar models. To obtain a reliable estimate of the spatial distribution of erosion and sediment transport requires a calibration and validation by means of spatially distributed data of soil loss, which in turn allows a calibration of spatially distributed parameters concerning transport capacity.

Palabras clave: erosión del suelo, transporte de sedimentos, inventarios de $\mathrm{Cs}^{137}$, modelo WATEM/SEDEM, coeficiente de capacidad de transporte, cuenca experimental de Arnás, Pirineo Central Español.

Key words: soil erosion, sediment transport, ${ }^{137} \mathrm{Cs}$ inventories, model WATEM/SEDEM, transport capacity coefficient, experimental Arnás catchment, Central Spanish Pyrenees.

Enviado el 2 de octubre de 2012

Aceptado el 15 de enero de 2013

*Correspondencia: Geoinformática, División Multidisciplinaria de la UACJ en Cuauhtémoc, Universidad Autónoma de Ciudad Juárez, 31579 Chihuahua, México. E-mail: luis.alatorre@uacj.mx

\section{Introducción}

En la actualidad, la atención científica y el destino de recursos financieros para el desarrollo de proyectos de investigación se están orientando cada vez más hacia una mejor comprensión y predicción de las consecuencias del cambio global sobre la erosión y la dinámica en la producción de sedimentos. El éxito de estas predicciones está en parte determinado por el desarrollo de nuevas herramientas: así, la incorporación de modelos de erosión y transporte de sedimentos a los Sistemas de Información Geográfica (SIG) hace que sea relativamente fácil explorar el creciente número de escenarios de cambio global, ya que permite evaluar espacialmente la ocurrencia de erosión y transporte de sedimentos, analizar su evolución temporal, y simular los efectos de los cambios en las variables climáticas y/o de los usos del suelo.

Una gran parte de los modelos de erosión aplicados a escala de cuenca se basan en una representación espacialmente agregada, es decir en la subdivisión del espacio en superficies a las que se supone un comportamiento homogéneo (subcuencas, unidades hidrológicas, etc.). Esta aproximación conlleva una deseable simplificación de los cálculos, pero presenta dificultades para abordar problemas relacionados con el transporte de sedimentos y la identificación de áreas fuente de sedimento, que deben tratarse mediante una representación espacial continua. Aunque la mayoría de los procesos de erosión y sedimentación se han estudiado en detalle mediante el uso de dispositivos experimentales, la evaluación de la relación entre la erosión del suelo in situ y la producción total de sedimentos a la salida de una cuenca es a menudo problemática debido a las dificultades asociadas a la conservación de un balance de masas en un sistema matemático en cascada. 
Los modelos espacialmente distribuidos posibilitan una aproximación más precisa a este problema, a costa de una mayor complejidad de cálculo (Merrit et al., 2003). Por otra parte el proceso de calibración y validación de un modelo espacialmente distribuido requiere datos reales de tasas de erosión y sedimentación espacialmente distribuidos, lo cual resulta muy costoso y, a veces, prácticamente imposible. En el mejor de los casos se llega a contar con datos de transporte de sedimentos en los principales ríos de la cuenca, si bien éstos rara vez abarcan un largo período de tiempo. Una metodología alternativa ha sido la utilización de tasas de erosión del suelo derivados de los registros sedimentológicos en embalses o lagos (p. ej., de Vente et al., 2008). Ello ha permitido predecir la producción total de sedimentos para el conjunto de cuencas que drenan a embalse, pero se ha sacrificado la capacidad de predecir espacialmente la redistribución del suelo en las laderas. En definitiva, la falta de datos espacialmente distribuidos de tasas de erosión y sedimentación es un problema importante que dificulta el uso de modelos de erosión espacialmente distribuidos, e incluso hace que la calibración del modelo sea prácticamente imposible de llevar a cabo (Alatorre et al., 2010).

Las dificultades asociadas con las técnicas clásicas para la estimación de la erosión han promovido el desarrollo de nuevos métodos. En las últimas décadas, las mediciones en campo de la lluvia radiactiva del $\mathrm{Cesio}^{137}\left(\mathrm{Cs}^{137}\right)$, subproducto de ensayos nucleares, ha permitido establecer inventarios que han sido utilizados para determinar las tasas de redistribución del suelo en puntos específicos en el paisaje. La redistribución del suelo se refiere al resultado neto de la erosión y sedimentación durante un período de aproximadamente 50 años (Walling y Quine, 1990). El uso de radioisótopos ha sido objeto de un creciente interés como un enfoque alternativo al análisis de la erosión del suelo, y se ha aplicado con éxito en una amplia gama de entornos (p. ej., Ritchie y McHenry, 1990; Walling y Quine, 1991; Walling y He, 1999; Collins et al., 2001; Navas, 2002; Bujan et al., 2003). Las estimaciones de tasas de redistribución del suelo a partir de inventarios de $\mathrm{Cs}^{137}$ se relacionan con una superficie pequeña en la que se obtiene la muestra de suelo (normalmente unos pocos $\mathrm{dm}^{2}$ ), y se toman a menudo como estimaciones puntuales cuando se considera a escala de cuenca. Generalmente, el estudio de los patrones espaciales de redistribución del suelo utilizando estimaciones puntuales de inventarios de $\mathrm{Cs}^{137}$ ha consistido en desarrollar una muestra suficientemente grande y realizar una interpolación espacial. Las tasas de redistribución de suelos derivados de inventarios de $\mathrm{Cs}^{137}$ también se han utilizado para validar los resultados de los modelos de erosión, incluyendo: i) Modelos empíricos de erosión tales como la Ecuación Universal de Pérdida de Suelo (RUSLE) (Ferro et al., 1998; López-Vicente et al., 2008); ii) Modelos espacialmente semi-distribuidos de erosión, como ANSWERS y AGNPS (De Roo, 1993; Walling et al., 2003), y iii) modelos completamente de base física y espacialmente distribuidos, como LISEM y WATEM/SEDEM (Takken et al., 1999; Feng et al., 2010).

El principal objetivo del presente estudio ha consistido en un análisis comparativo de los resultados obtenidos en el proceso de calibración y validación de WATEM/SEDEM, un modelo empírico-conceptual espacialmente distribuido para predecir erosión y la producción de sedimentos en dos cuencas del Pirineo aragonés : i) la cuenca del embalse de Barasona $\left(1504 \mathrm{~km}^{2}\right)$, drenada por el Río Ésera e Isábena, donde, 
para el proceso de calibración se utilizó el registro de 3 años de sedimentos en suspensión (mayo 2005-mayo 2008) a la salida de la subcuenca del Río Isábena y, para la validación, se utilizó el registro histórico de sedimentación del embalse de Barasona; y, ii) la cuenca experimental de Arnás $\left(2.84 \mathrm{~km}^{2}\right)$, donde se contó para la calibración con datos espacialmente distribuidos de tasas de erosión y sedimentación derivadas de inventarios de $\mathrm{Cs}^{137}$, más el registro de siete años de sedimento en suspensión a la salida de la cuenca de Arnás para el proceso de validación. Se describen los compromisos adoptados en la calibración del modelo, y se discute la validez de los resultados y su aplicación. El enfoque seguido es transferible a otras regiones del mundo.

\section{Materiales y métodos}

\subsection{WATEM/SEDEM}

El modelo WATEM/SEDEM se utiliza para estimar los flujos de sedimento desde las laderas a la red de drenaje. Una descripción más detallada del modelo puede encontrarse en Van Oost et al. (2000), Van Rompaey et al. (2001a) y Verstraeten et al. (2002); en este trabajo sólo se aporta una descripción de los principios básicos.

WATEM/SEDEM es un modelo empírico-conceptual espacialmente distribuido que utiliza mapas ráster para el cálculo de la erosión y producción de sedimentos. En este caso los mapas fueron generados en IDRISI. En un primer paso el modelo calcula la erosión anual potencial píxel por píxel, basada en la ecuación de la RUSLE en 2D (Renard et al., 1991):

$$
E=R K L S_{2 D} C P(1)
$$

donde $E$ es la pérdida de suelo media anual $\left(\mathrm{kg} \mathrm{m}^{-2}\right.$ año $\left.{ }^{-1}\right), R$ es el factor de erosividad de la precipitación (MJ mm m${ }^{-2} \mathrm{~h}^{-1} \mathrm{año}^{-1}$ ), $L S_{2 D}$ es el factor de longitud de ladera (Desmet y Govers, 1996) que toma en cuenta la concentración de la escorrentía en una topografía compleja del paisaje en 2D, $C$ es el factor adimensional de manejo de cultivos y $P$ el factor de práctica de conservación del suelo.

En un segundo paso WATEM/SEDEM conduce todos los sedimentos generados ladera abajo hasta llegar a un píxel del cauce principal. Los sedimentos sobre la ladera son transportados por un flujo superficial de acuerdo a la ecuación de capacidad de transporte (Van Rompaey et al., 2001a):

$$
T C=k t c R K\left(L S_{2 D}-4.1 s^{0.8}\right)
$$

donde $T C$ es la capacidad de transporte $\left(\mathrm{kg} \mathrm{m}^{-1}\right.$ año $\left.{ }^{-1}\right), k t c(\mathrm{~m})$ es el coeficiente empírico de capacidad de transporte que depende del uso y cobertura de suelo, y $s$ es la pendiente $\left(\mathrm{m} \mathrm{m}^{-1}\right)$. El parámetro ktc representa la longitud de pendiente necesaria para producir una cantidad de sedimento igual al sedimento que se produciría en un suelo desnudo con idéntica pendiente (Verstraeten, 2006). En una aplicación previa de WATEM/SEDEM se encontró una diferencia significativa entre suelo arable (altamen- 
te propenso a la erosión) y suelo con baja propensión a la erosión, incluyendo pastizales y bosques (Van Rompaey et al., 2001a, 2005; Verstraeten et al., 2007). Además, este factor depende de la escala (i.e., el tamaño del píxel) por lo que necesita ser calibrado para cada aplicación del modelo.

Para cada píxel, WATEM/SEDEM calcula un balance de masas, donde el sedimento de entrada más el sedimento generado en el píxel es transportado ladera abajo (si éste es más bajo que la capacidad de transporte). Existen otras propuestas para modelizar la capacidad de transporte $T C$ : por ejemplo, Verstraeten et al. (2007) propusieron una ecuación específica para zonas donde la erosión en cárcavas es dominante. En este estudio se ha utilizado la formulación original, tal y como se aplica en la versión WATEM/SEDEM 2004.

\section{2. Áreas de estudio y base de datos}

\subsubsection{Cuenca del embalse de Barasona}

La cuenca del embalse de Barasona está drenada por dos ríos: el río Ésera y, su mayor tributario, el río Isábena (Fig. 1A). La cuenca cubre una superficie de $1504 \mathrm{~km}^{2}$ (1066 km² y $438 \mathrm{~km}^{2}$ respectivamente) y se localiza en el Pirineo Central español; su desembocadura se sitúa en las coordendas $42^{\circ} 3^{\prime} \mathrm{N}, 0^{\circ} 16^{\prime} \mathrm{W}$ y $42^{\circ} 42^{\prime} \mathrm{N}, 0^{\circ} 42^{\prime} \mathrm{W}$. La cabecera de la cuenca $\left(12.9 \mathrm{~km}^{2}\right)$ se ha descontado como área de contribución efectiva debido a que drena por vía subterránea hacia el río Garona, en la vertiente norte del Pirineo; esto representa un promedio de descarga de aproximadamente $3 \mathrm{~m}^{3} \mathrm{~s}^{-1}$ desde el río Ésera (López-Moreno et al., 2002). La cuenca se caracteriza por fuertes contrastes topográficos, edáficos y de usos de suelo y de cubierta vegetal (Alatorre y Beguería, 2009a, b). El rango altitudinal está comprendido entre 450 m s.n.m. en la parte sur y 3404 m s.n.m. en la parte norte del río Ésera o 2720 m s.n.m. en la parte norte del río Isábena (Fig. 1B).

La litología del área de estudio (Fig. 1C) comprende unidades geológicas organizadas en varias unidades geoestructurales con orientaciones ONO-ESE: i) el Pirineo Axial, que está compuesto por rocas paleozoicas (cuarcitas, calizas y lutitas) y granodioritas, con cumbres por encima de los 3000 m s.n.m.; ii) las Sierras Interiores, que forman un enorme pliegue con cabalgamiento de los sedimentos cretácicos y paleógenos formados principalmente por calizas y areniscas, lo que se traduce en un relieve escarpado; iii) la Depresión Intermedia, constituida principalmente por materiales con alta propensión a ser erosionadas (margas eocenas), dando un relieve relativamente suave; iv) las Molasas Prepirenaicas, compuestas por sedimentos continentales del oligoceno (conglomerados y areniscas), dando un relieve abrupto y divisorias suaves; y v) las Sierras Exteriores, formadas por calizas en la parte sur de la cuenca.

El mapa de tipos de suelos (Fig. 1D), basado en la cartografía realizada por Machín (1990), refleja la importancia de los suelos minerales con poco desarrollo (incluyendo regosoles, leptosoles y fluvisoles), que ocupan el 53\% de la superficie, y los suelos con 

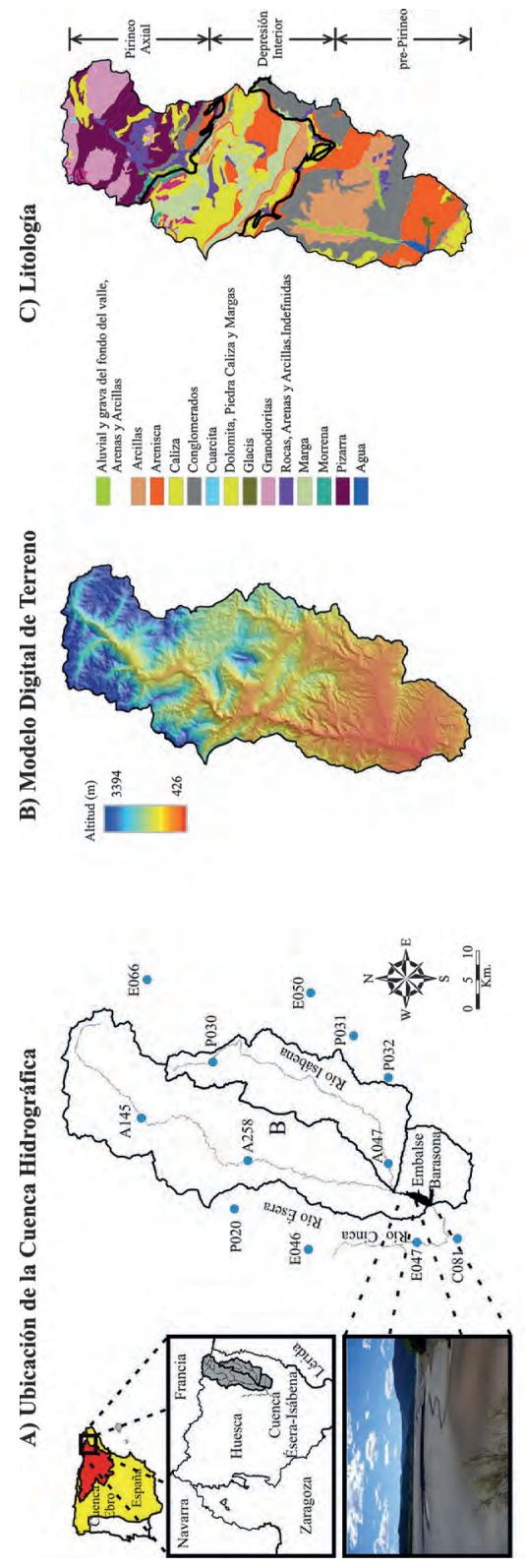

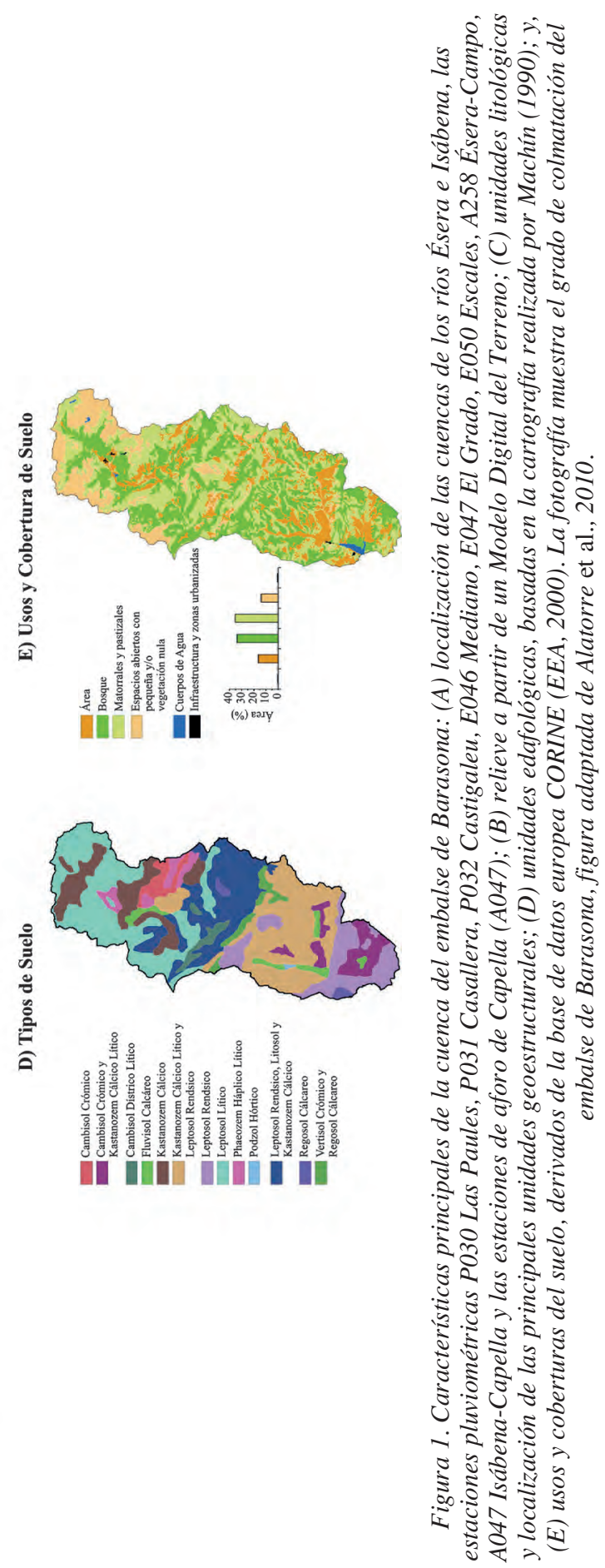


una notable acumulación de materia orgánica, incluyendo kastanozems, que ocupan el $32 \%$ de la superficie

El clima es típico de montaña, relativamente húmedo y frío, con influencias del océano Atlántico y del mar Mediterráneo (García-Ruiz et al., 2001). Además presenta un fuerte gradiente $\mathrm{N}-\mathrm{S}$ : el rango de precipitación media anual y el rango de temperaturas está comprendido entre los $2000 \mathrm{~mm}_{\text {año }}^{-1}$ y $4^{\circ} \mathrm{C}$ en la cabecera y por debajo de

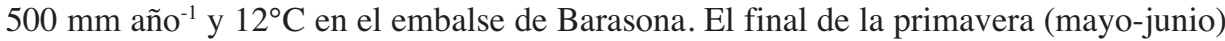
y el otoño (noviembre), seguidos del verano, son los periodos más húmedos. Las tormentas son frecuentes y las más intensas ocurren en el verano y el otoño. La presencia de nieve ocurre desde mediados de noviembre a mayo por encima de los $1700 \mathrm{~m}$ s.n.m. (Chueca y Julián, 2002). Las avenidas más importante tienden a ocurrir en mayo-junio y ocasionalmente en otoño (López-Moreno y García-Ruiz, 2004). El mapa de usos y coberturas de suelo (Fig. 1E) se ha derivado de la base de datos Europea CORINE (EEA, 2000). El área esta compuesta principalmente por matorrales y pastizales (36\%), bosques $(32 \%)$, cultivos $(16 \%)$ y áreas con poca o nula vegetación $(14 \%)$.

El embalse de Barasona fue construido en el año 1932 para abastecer zonas de regadío y para generar electricidad. La capacidad inicial de almacenamiento era de $71 \times 10^{6} \mathrm{~m}^{3}$. En el año 1972 la altura de la presa se incrementó y la capacidad de almacenamiento aumentó a $92.71 \times 10^{6} \mathrm{~m}^{3}$. Actualmente el embalse tiene un área de inundación de 692 ha, una profundidad máxima de $60 \mathrm{~m}$, y una profundidad media de $16.5 \mathrm{~m}$. La capacidad de la estación hidroeléctrica es de $26 \mathrm{MW}$. El Canal de Aragón y Cataluña, a la salida del embalse, riega 104820 ha de tierras de cultivo. La temporada de riego se extiende desde marzo a octubre, con una demanda máxima desde mayo a agosto. Al final de la temporada de riego y antes de las precipitaciones del otoño y del invierno, el agua almacenada en el embalse llega a su nivel mínimo, dejando al descubierto los sedimentos acumulados en el fondo del embalse (véase la fotografía, Fig. 1A). La capacidad de almacenamiento del embalse ha disminuido considerablemente en los últimos 65 años (Avendaño et al., 1997a, b; Navas et al., 1998; Valero-Garcés et al., 1999). Durante la década de 1950 el reservorio fue drenado desde el fondo hacia la salida, de manera que la mayoría de los sedimentos depositados fueron exportados fuera del embalse debido a la energía cinética del caudal (Autoridades de la cuenca del río Ebro-CHE, técnicos y personal de comunicación). Debido al mal funcionamiento del drenado de fondo después de la década de 1950 el régimen de gestión cambió y éste ya no fue aplicado. Un estudio sísmico en 1995 indicó que el embalse había perdido aproximadamente un tercio de su capacidad inicial de almacenamiento y el volumen de los sedimentos acumulados era de $16-18 \times 10^{6} \mathrm{~m}^{3}$, con un espesor máximo de 20-25 m cerca de la presa (Sanz-Montero et al., 1996). Tras años de sedimentación, la toma del embalse se colmató y se procedió a una operación que implicó, durante tres años consecutivos (1995-1998), el retiro de los sedimentos después de la temporada de riego. Sin embargo, la mayor parte de los sedimentos acumulados no se vio afectada por estas operaciones. A partir de técnicas batimétricas, Sanz-Montero et al. (1996) estimaron una producción específica de sedimentos para la cuenca del embalse de Barasona de $3.50 \mathrm{Mg} \mathrm{ha}^{-1} \mathrm{año}^{-1}$, un valor similar a los valores observados en otras cuenca del Pirineo (Almorox, 1994). 
Tabla 1. Características principales de los suelos en la cuenca de Arnás, diferenciando entre las dos laderas (media \pm desviación estándar sobre el perfil del suelo), tabla adaptada de Navas et al. (2005).

\begin{tabular}{|l|r|r|}
\hline & Ladera suroeste, Bosques $(\mathbf{n = 4 8})$ & Ladera noreste, Matorral $(\mathbf{n = 2 9})$ \\
\hline $\mathrm{pH}$ & $7.97( \pm 0.42)$ & $8.17( \pm 0.19)$ \\
\hline Arcillas $\left(\mathrm{g} \mathrm{kg}^{-1}\right)$ & $210( \pm 31)$ & $195( \pm 34)$ \\
\hline Limos $\left(\mathrm{g} \mathrm{kg}^{-1}\right)$ & $660( \pm 63)$ & $620( \pm 73)$ \\
\hline Arena $\left(\mathrm{g} \mathrm{kg}^{-1}\right)$ & $130( \pm 85)$ & $180( \pm 103)$ \\
\hline Materia orgánica $\left(\mathrm{g} \mathrm{kg}^{-1}\right)$ & $59( \pm 22)$ & $54( \pm 25)$ \\
\hline Densidad $\left(\mathrm{g} \mathrm{kg}^{-1}\right)$ & $1.12( \pm 1.22)$ & $1.19( \pm 0.61)$ \\
\hline Humedad $(\%)$ & $17( \pm 6.7)$ & $11( \pm 7.7)$ \\
\hline Porosidad $(\%)$ & $57( \pm 5.9)$ & $55( \pm 6.2)$ \\
\hline
\end{tabular}

\subsubsection{Cuenca experimental de Arnás}

La cuenca experimental de Arnás se localiza en el valle de Borau, en la cabecera de la cuenca del río Aragón en el Pirineo Central (Fig. 2A). Tiene una superficie de 2.84 $\mathrm{km}^{2}$ y un rango altitudinal comprendido entre 912 y 1339 m s.n.m. (Fig. 2B y 2C). El clima es sub-mediterráneo con influencia Atlántica, la temperatura media anual es de $10^{\circ} \mathrm{C}$ y la precipitación media anual de $930 \mathrm{~mm}$ para el periodo de octubre de 1996 a septiembre de 2009. La precipitación es más abundante en otoño y primavera, si bien en el verano las tormentas de tipo convectivo pueden ser relativamente frecuentes. La isoterma $0^{\circ} \mathrm{C}$ para el periodo noviembre-abril se sitúa alrededor de $1650 \mathrm{~m}$ de altitud por lo que los episodios de nieve no son habituales.

La cuenca se asienta sobre un flysch del eoceno, es decir, sobre capas alternadas de margas y areniscas. Las dos laderas de la cuenca presentan contrastadas características fisiográficas. En la ladera con orientación suroeste, con una pendiente media de $0.5 \mathrm{~m} \mathrm{~m}^{-1}$, encontramos suelos poco desarrollados, Leptosoles réndsicos y Regosoles calcáreos, sobre materiales poco consolidados (Navas et al., 2005). Sobre esta ladera pueden apreciarse deslizamientos superficiales todavía activos en la actualidad, aunque quedan desconectados de la red de drenaje (Lorente et al., 2000) por lo que tienen una escasa influencia en la producción de sedimentos a escala de cuenca (Bathurst et al., 2007). En la ladera umbría, de pendiente más suave $\left(0.28 \mathrm{~m} \mathrm{~m}^{-1}\right)$, los suelos son Kastanozems háplicos y Phaeozems. Estos suelos son mas desarrollados y profundos $(50 \mathrm{a}>75 \mathrm{~cm})$ con horizontes claramente diferenciados (Navas et al., 2005). Antiguas lenguas de movimientos en masa profundos dan un aspecto corrugado a esta ladera. Los rellanos del fondo del valle cuentan con pendientes inferiores a $0.09 \mathrm{~m} \mathrm{~m}^{-1}$ correspondiendo, algunos de ellos, a pequeños restos de terrazas fluviales. Predominan los Fluvisoles calcáreos, suelos profundos y desarrollados sobre depósitos aluviales, con una mínima diferenciación entre horizontes (Navas et al., 2005). Las principales propiedades de los suelos se muestran en la tabla 1. 


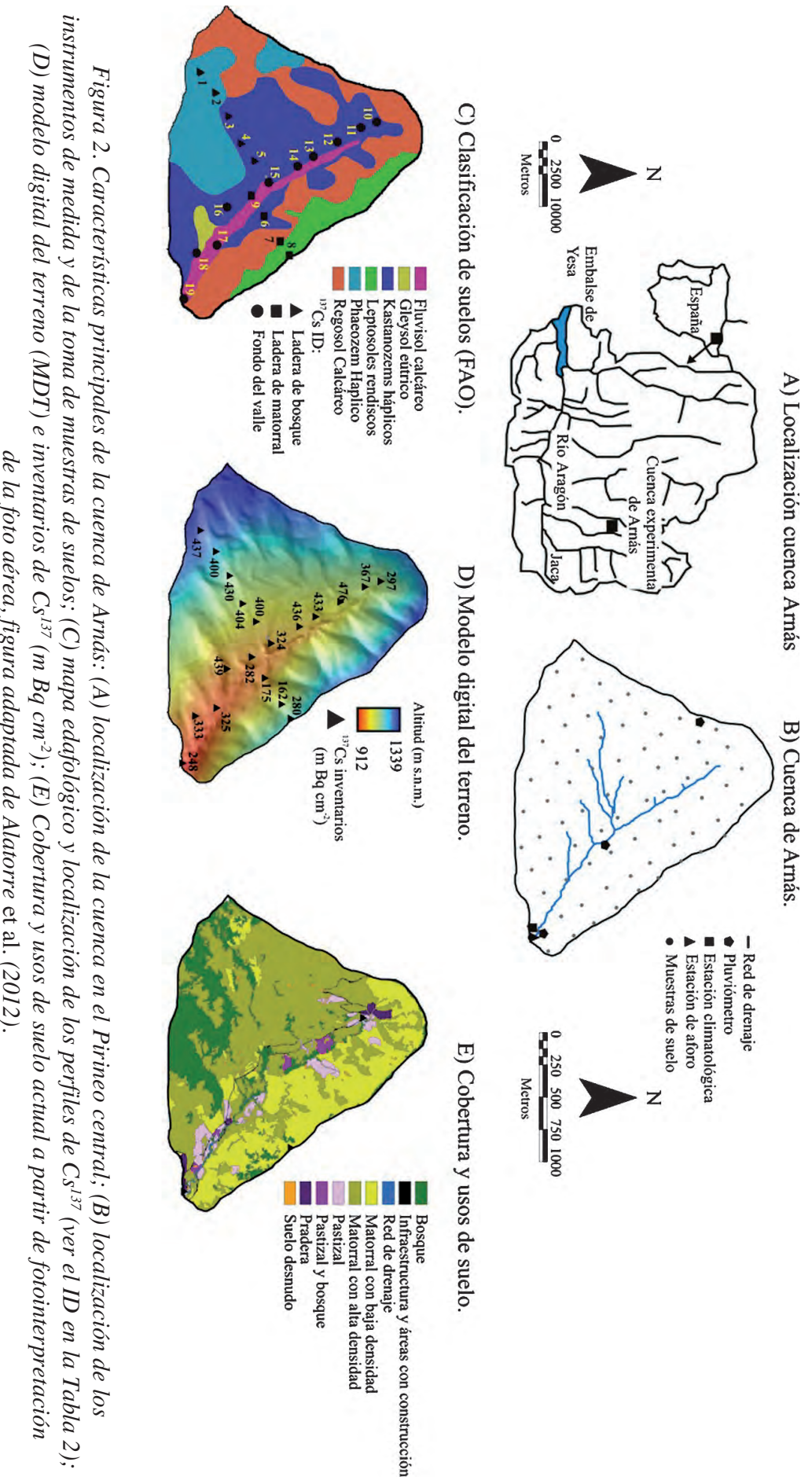


En la ladera suroeste (ladera de matorral) predomina el matorral mediterráneo (Buxus sempervirens, Genista scorpius), y en la ladera noreste (ladera de bosque) un matorral más denso (Juniperus communis, Buxus sempervirens, Echynospartum horridum) y grandes manchas de bosque de Pinus sylvestris (Fig. 2E).

Hasta mediados del siglo XX la cuenca de Arnás estuvo totalmente cultivada por campos en pendiente. En el Pirineo, las laderas solana se cultivaban hasta una altitud de 1600 m s.n.m. mientras que las laderas umbría no se solían cultivar (García-Ruiz y Lasanta, 1990). Excepcionalmente, en la cuenca de Arnás también se cultivó la ladera noreste debido a su suave pendiente que permitía una insolación relativamente importante y suficiente para el cultivo de cereales. En la ladera suroeste, las zonas cóncavas estaban ocupadas por terrazas abancaladas, mientras que las laderas convexas y rectas se cultivaron bajos sistemas de agricultura nómada con escasa prácticas de conservación de suelos (Lasanta et al., 2006). Desde principios del siglo XX, los campos de cultivo fueron abandonados progresivamente, empezando por los peores campos utilizados por una agricultura nómada. En la década de 1950 prácticamente todas las laderas de la cuenca estaban abandonadas. Los fondos de valle se abandonaron con posterioridad, durante la década de 1970. Como consecuencia del abandono de las tierras de cultivo ocurrió un complejo proceso de colonización vegetal, con una progresiva entrada de comunidades arbustivas densas y una presencia cada vez mayor de árboles. Actualmente, muchos de los campos de los fondos de valle todavía se mantienen como pastos, si bien muchos de ellos también se ven afectados por la colonización de Genista scorpius debido a la baja presión del pastoreo. Este proceso descrito es similar al observado en otras regiones de Europa, en la que los procesos de colonización vegetal son consecuencia del abandono de la actividad agrícola (Kozak, 2003; Taillefumier y Piégay, 2003; Torta, 2004).

La cuenca de Arnás forma parte de la red de cuencas experimentales del Instituto Pirenaico de Ecología (CSIC) y ha sido objeto de numerosos trabajos con el fin de entender el comportamiento hidrogeomorfológico de un ambiente de campos abandonados. Así, se ha estudiado su funcionamiento hidrológico (García-Ruiz et al., 2005; LanaRenault et al., 2007a, b), las propiedades físico-químicas de los suelos (Navas et al., 2002a, b, 2005, 2008; Seeger et al., 2004) y los procesos erosión y transporte de sedimentos (Lana-Renault y Regüés, 2007, 2009; López-Vicente et al., 2011).

\subsection{Parametrización de WATEM/SEDEM}

Para la cuenca del embalse de Barasona y para la cuenca experimental de Arnás se preparó una base de datos en formato ráster, con una resolución espacial de 20 x $20 \mathrm{~m}$ y 5 x $5 \mathrm{~m}$ respectivamente. Para derivar la red de drenaje principal se empleó un Modelo Digital del Terreno (MDT) y el establecimiento de un umbral de $1 \mathrm{~km}^{2}$ para el área de contribución. También se prepararon mapas de: i) usos de suelo; ii) Factor-K, erodibilidad del suelo; iii) Factor-R, erosividad de la lluvia; y, iv) Factor-C, cobertura del suelo (Figs. 3 y 4, respectivamente). La información detallada sobre el desarrollo de este conjunto de datos se proporciona en Alatorre et al. $(2010,2012)$. 


\section{A) Usos de Suelo}

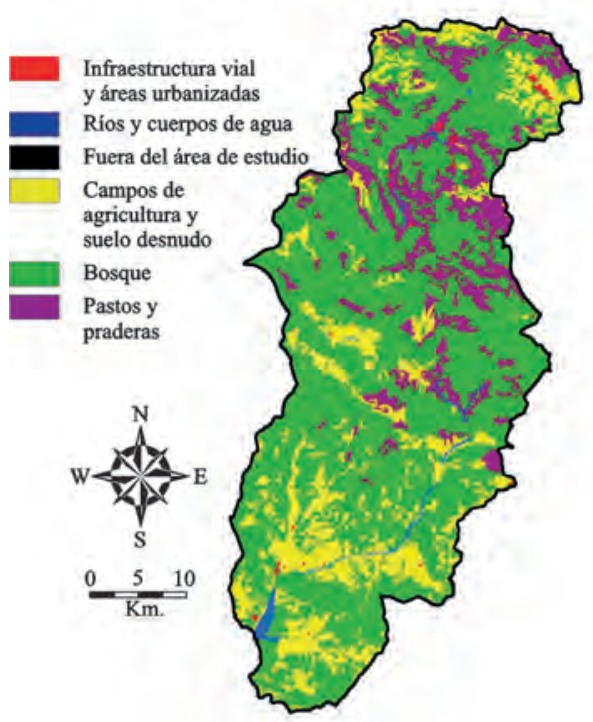

C) Factor-R

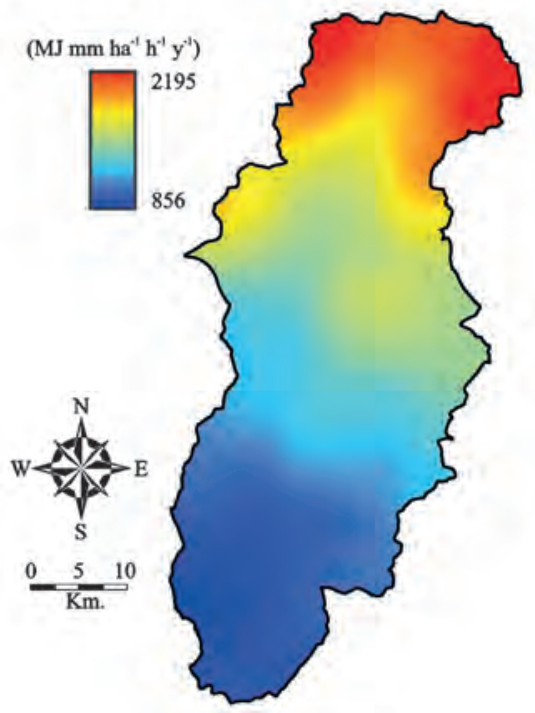

\section{B) Factor-K}

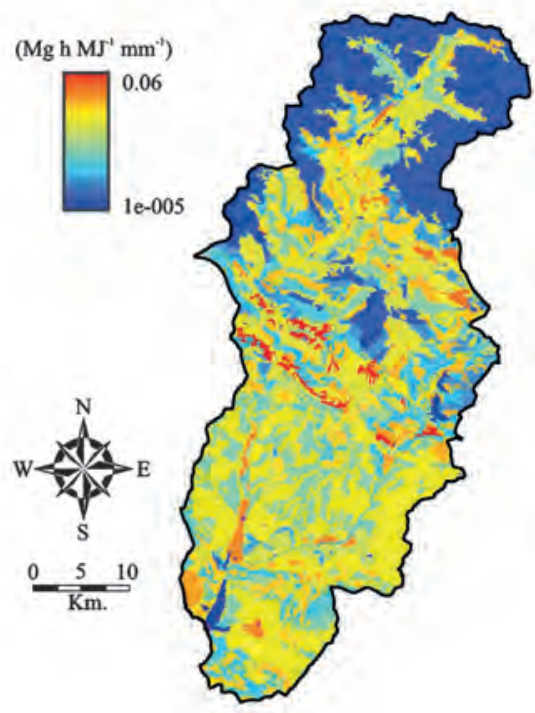

D) Factor-C

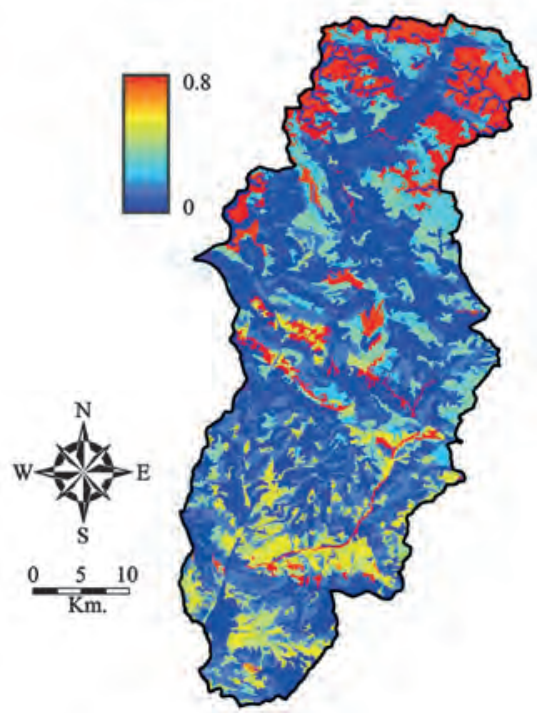

Figura 3. Parámetros de entrada al modelo WATEM/SEDEM para su aplicación en la cuenca del embalse de Barasona: (A) mapa de usos y cobertura de suelos, derivado de la base de datos Europea de coberturas CORINE (EEA, 2000); (B) mapa del factor de erodibilidad del suelo (Factor-K, RUSLE, Mg h MJ $\mathrm{JJ}^{-1} \mathrm{~mm}^{-1}$ ); (C) mapa de la erosividad de la precipitación (R-factor in RUSLE, MJ mm ha $a^{-1} h^{-1}$ año-1); y, (D) mapa del factor de cobertura de suelo

(Factor-C, RUSLE), figura adaptada de Alatorre et al. (2010). 
A) Relieve sombreado de la red de drenaje.

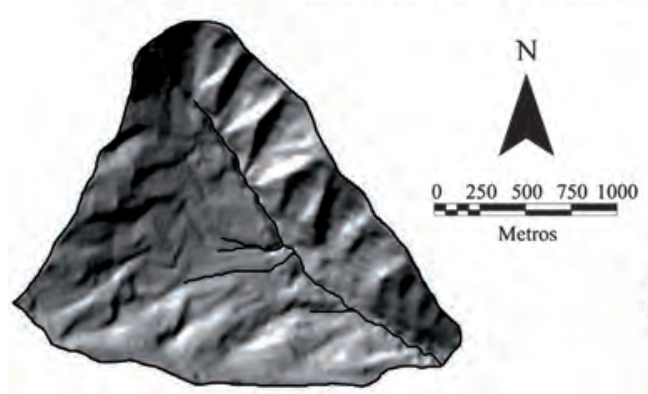

C) Factor- $\mathrm{K}\left(\mathrm{Mg} \mathrm{h} \mathrm{MJ} \mathrm{mm}^{-1}\right)$.

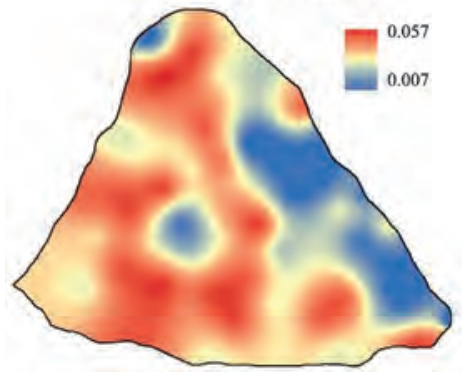

B) Usos de Suelo.

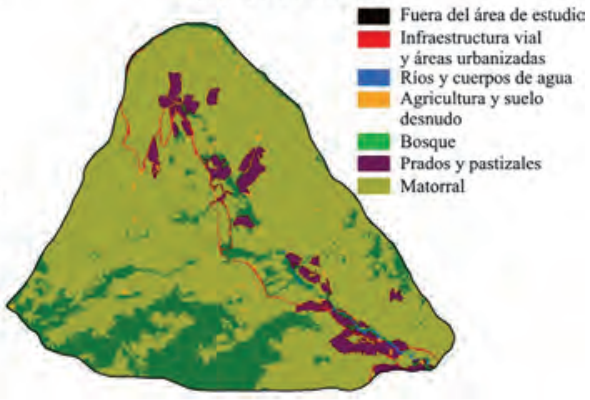

D) Factor-C

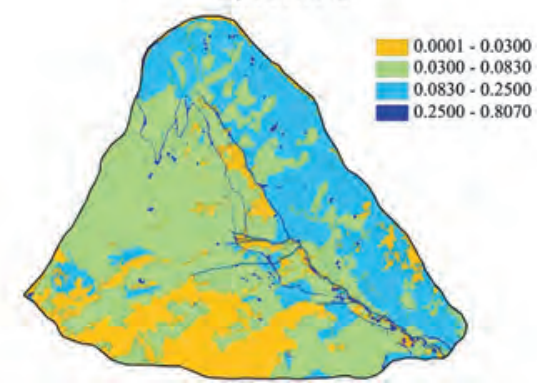

Figura 4. Parámetros de entrada al modelo WATEM/SEDEM para su aplicación en la cuenca de Arnás: (A) mapa de la red de drenaje derivado del MDT usando un área de contribución de $1 \mathrm{~km}^{2}$ (línea continua); (B) mapa de cobertura y usos de suelos, derivado del mapa de cobertura y usos de suelo actual; $(C)$ mapa del factor de erodibilidad del suelo (Factor-K de la RUSLE, Mg h MJ $\mathrm{Jm}^{-1}$ ); y, (D) mapa del factor de cobertura de suelo (Factor-C de la RUSLE), figura adaptada de Alatorre et al. (2012).

\subsection{Calibración de WATEM/SEDEM}

La calibración del modelo WATEM/SEDEM requiere encontrar un valor óptimo para el coeficiente de capacidad de transporte máximo $\left(\mathrm{ktc}_{\max }\right)$, relativo a usos agrícolas, y mínimo $\left(\mathrm{ktc}_{\min }\right)$, relativo a áreas con cubierta forestal densa. Se recomienda que el cociente $\mathrm{ktc}_{\max } / \mathrm{ktc}_{\min }$ permanezca constante durante el proceso de calibración para poderlo considerar como un solo parámetro (Verstraeten, 2006). En la mayoría de los casos la calibración se realiza a partir de datos de producción de sedimentos a la salida de la cuenca de estudio. El modelo original fue calibrado usando datos observados de producción de sedimentos en 21 cuencas al sur de Flandes, Bélgica (Van Rompaey et al., 2001a; Verstraeten y Poesen, 2001), donde se encontraron valores de $\mathrm{ktc}_{\min }=42 \mathrm{~m}$ en suelos no cultivados y $\mathrm{ktc}_{\max }=75 \mathrm{~m}$ para campos de cultivo. En estudios posteriores, los mismos autores encontraron valores óptimos de $\mathrm{ktc}_{\min }=75 \mathrm{~m} \mathrm{y} \mathrm{ktc}_{\max }=250 \mathrm{~m}$ (Verstraeten et al., 2006). No es de sorprender que los valores de ktc presenten valores tan distantes de un estudio a otro ya que, según Van Rompaey et al. (2001a), 
WATEM/SEDEM sólo puede ser calibrado para una combinación específica de tamaño de píxel y de elección del método de transporte de sedimento. El uso de distintos métodos de transporte de sedimentos y/o tamaño de píxel da como resultado valores óptimos de calibración diferentes.

Para la calibración de $\mathrm{ktc}_{\max } \mathrm{y} \mathrm{ktc}_{\min }$ en la cuenca de los ríos Ésera-Isábena se utilizó el registro de sedimentos en suspensión de 3 años (mayo 2005-mayo 2008) de la estación de aforo de Capella (López-Tarazón et al., 2009), localizada a la salida de la cuenca del río Isábena. Para cada uno de los 3 años se calculó el Factor-R (937, 2180 y1900 MJ mm ha ${ }^{-1} \mathrm{~h}^{-1}$ respectivamente) de acuerdo con los registros de precipitación disponibles en el área de estudio. Se calculó la producción anual de sedimentos modificando los valores de $\mathrm{ktc}_{\max } \mathrm{y} \mathrm{ktc}_{\min }$. Para cada combinación de $\mathrm{ktc}_{\max } \mathrm{y} \mathrm{ktc}_{\min }$ se obtuvo una predicción para cada uno de los años, permitiendo comparar los valores predichos por WATEM/SEDEM con los registrados en la estación de aforo.

En la cuenca experimental de Arnás se utilizó la base de datos espacialmente distribuida de 19 inventarios de $\mathrm{Cs}^{137}$, localizados a lo largo de tres transectos representativos de la cuenca: i) 5 puntos localizados en la ladera suroeste (bosque); ii) 4 puntos en la ladera noreste (matorral); y iii) 10 puntos a lo largo del fondo del valle (Tabla 2 y Fig. 2C). Las tasas de redistribución de suelo fueron calculadas en cada uno de los puntos y se compararon con los inventarios de referencia de $\mathrm{Cs}^{137}$, los cuales corresponden con valores promedio para el periodo comprendido entre 1963 (inicio de la precipitación de $\mathrm{Cs}^{137}$ en la región) y 2003 (momento de la toma de muestras y análisis del radioisótopo). Remitimos al lector interesado a consultar el artículo de Navas et al. (2005) donde se presentan el análisis e interpretación detallados de los datos de $\mathrm{Cs}^{137}$.

Para determinar el grado de ajuste del modelo se aplicó en ambas cuencas el estadístico de eficiencia Nash-Sutcliffe (NS) (Nash y Sutcliffe, 1970) de acuerdo con la siguiente ecuación:

$$
N S=1-\frac{\sum_{i=1}^{n}\left(O_{i}-P_{i}\right)^{2}}{\sum_{i=1}^{n}\left(O_{i}-O_{\text {ined }}\right)^{2}}
$$

donde $n$ es el número de observaciones, $O_{i}$ es el valor observado, $O_{\text {med }}$ es el valor medio de los valores observados, y $P_{i}$ es el valor predicho. $N S$ puede variar desde $-\infty$ a 1 , de manera que un valor de NS próximo a 1 representa una mayor eficiencia del modelo. También se utilizó la raíz del error cuadrático medio (RRMSE) de acuerdo con la siguiente ecuación:

$$
\text { RRMSE }=\mathrm{I}-\frac{\sqrt{\frac{1}{n} \sum_{i=1}^{n}\left(O_{i}-P_{i}\right)^{2}}}{\frac{1}{n} \sum_{i=1}^{n} O_{i}}
$$


Tabla 2. Inventarios de $C^{137}$ y tasas de redistribución de suelos para el periodo 1963-2003 a lo largo de tres transectos en la cuenca experimental de Arnás (Navas et al., 2005). Los valores positivos indican valores netos de erosión y los negativos indican valores netos de sedimentación. La localización de los inventarios de Cs ${ }^{137}$ se muestra en la Figura $2 C$.

\begin{tabular}{|c|c|c|c|}
\hline Transecto & ID del punto & $\begin{array}{r}{ }^{137} \mathrm{Cs} \\
\left(\mathrm{m} \mathrm{Bq} \mathrm{cm}^{-2}\right)\end{array}$ & $\begin{array}{r}\text { Redistribución del suelo } \\
\left(\mathrm{Mg} \mathrm{ha}^{-1} \text { año }^{-1}\right)\end{array}$ \\
\hline Bosque & 1 & 437 & 0.9 \\
\hline Bosque & 2 & 400 & 0 \\
\hline Bosque & 3 & 430 & 0.8 \\
\hline Bosque & 4 & 404 & 0.1 \\
\hline Bosque & 5 & 400 & 0 \\
\hline Matorral & 6 & 175 & -26.4 \\
\hline Matorral & 7 & 162 & -29.5 \\
\hline Matorral & 8 & 280 & -11.6 \\
\hline Matorral & 9 & 282 & -14.3 \\
\hline Fondo del valle & 10 & 297 & -7.4 \\
\hline Fondo del valle & 11 & 367 & -2.0 \\
\hline Fondo del valle & 12 & 476 & 2.2 \\
\hline Fondo del valle & 13 & 433 & 1.0 \\
\hline Fondo del valle & 14 & 436 & 1.0 \\
\hline Fondo del valle & 15 & 324 & -4.3 \\
\hline Fondo del valle & 16 & 439 & 1.2 \\
\hline Fondo del valle & 17 & 325 & -5.2 \\
\hline Fondo del valle & 18 & 333 & -4.7 \\
\hline Fondo del valle & 19 & 248 & -44.6 \\
\hline
\end{tabular}

Para la cuenca del río Isábena, los valores de NS y RRMSE fueron calculados con la producción total de sedimentos $\left(\mathrm{Mg}\right.$ año $\left.{ }^{-1}\right)$ y la producción específica de sedimentos $\left(\mathrm{Mg} \mathrm{ha}^{-1} \mathrm{año}^{-1}\right)$ registrados en la estación de aforo de Capella. Para la cuenca experimental de Arnás, los valores de NS y RRMSE se calcularon con los valores de erosión neta predichos y observados en los puntos de los inventarios de referencia de $\mathrm{Cs}^{137}$.

\subsection{Validación de WATEM/SEDEM}

Una vez que se obtuvieron los valores de $\mathrm{ktc}_{\max } \mathrm{y} \mathrm{ktc}_{\text {min }}$ óptimos para la subcuenca del río Isábena, se procedió a aplicar WATEM/SEDEM a toda la cuenca del embalse de Barasona. Así se pudo comparar y validar los valores predichos por el modelo con el registro histórico de sedimentación en el embalse de Barasona. La validación en la cuenca experimental de Arnás se realizó para cada uno de los 7 años con registro de sedimentos a la salida de la cuenca, para el periodo coprendido entre octubre de 1999 y septiembre de 2008 (se excluyeron los años hidrológicos mayo de 2005 y julio de 2007 
debido a la falta de datos). La información detallada acerca de la instrumentación y el registro de los datos en la cuenca experimental de Arnás puede consultarse en el trabajo de Lana-Renault et al. (2009). También se utilizaron los datos de producción de sedimentos a la salida de la cuenca para comparar la calibración obtenida a partir de los inventarios de $\mathrm{Cs}^{137}$ (datos internos) con una calibración basada en la producción de sedimentos a la salida de la cuenca (datos externos).

Los resultados completos del proceso de calibración y validación en la cuenca del embalse de Barasona se pueden consultar en el trabajo de Alatorre et al. (2010). Éstos comprenden: i) las principales fuentes de sedimentos en relación a los usos y coberturas de suelo; ii) las principales fuentes de sedimentos en relación a las distintas unidades geoestructurales; iii) la comparación en orden de magnitud de la producción de sedimentos de cada una de las 23 subcuencas que forma el sistema; y iv) la relación de entrega de sedimentos y el área de cada una de estas subcuencas. Para la cuenca experimental de Arnás, los resultados detallados se pueden consultar en el trabajo de Alatorre et al. (2012) que incluye i) las principales fuentes de sedimentos en relación a los usos y coberturas de suelo y ii) la evaluación de dos escenarios alternativos de usos de suelo: un primer escenario que corresponde a principios del siglo $\mathrm{XX}$, cuando toda la cuenca estaba cultivada, y un segundo escenario futuro hipotético en el que se propone una densificación de la cubierta vegetal. En este trabajo sólo nos vamos a centrar en el proceso de calibración y validación para demostrar los inconvenientes que surgen al no disponer de una base de datos de tasas de erosión y sedimentación espacialmente distribuida.

\section{Resultados}

\subsection{Calibración de WATEM/SEDEM}

Los resultados del proceso de calibración utilizando los 3 años de producción de sedimentos registrados a la salida del río Isábena se presentan en la Fig. 5. Las gráficas en dos dimensiones muestran claramente el problema que se tiene al intentar calibrar dos parámetros $\left(\mathrm{ktc}_{\max } \mathrm{y} \mathrm{ktc}{ }_{\min }\right)$ con una sola variable (en este caso, los sedimentos registrados a la salida de la subcuenca). Los resultados evidencian que resulta imposible encontrar un solo conjunto de parámetros que optimicen la función de error. En cambio, se obtiene una serie de posibles combinaciones para los parámetros que producen resultados igualmente buenos, los cuales se representan como un "valle" en la distribución de los valores de RRMSE y como una "cresta" en la gráfica de NS. En el caso del río Isábena se emplearon datos para cada uno de los 3 años, los cuales pueden ser considerados como repeticiones experimentales, sin que esto afectara a la dificultad asociada a calibrar dos parámetros con solo una variable (producción de sedimentos a la salida de la subcuenca).

El problema planteado en la Fig. 5 obliga a reducir la calibración a una sola dimensión, mediante la combinación de ambos parámetros en uno sólo. Esto se hizo fijando una relación constante entre ambos parámetros, tal y como ya ha sido propuesto en otras aplicaciones de WATEM/SEDEM. Sin embargo, esto introduce un problema adicional debi- 

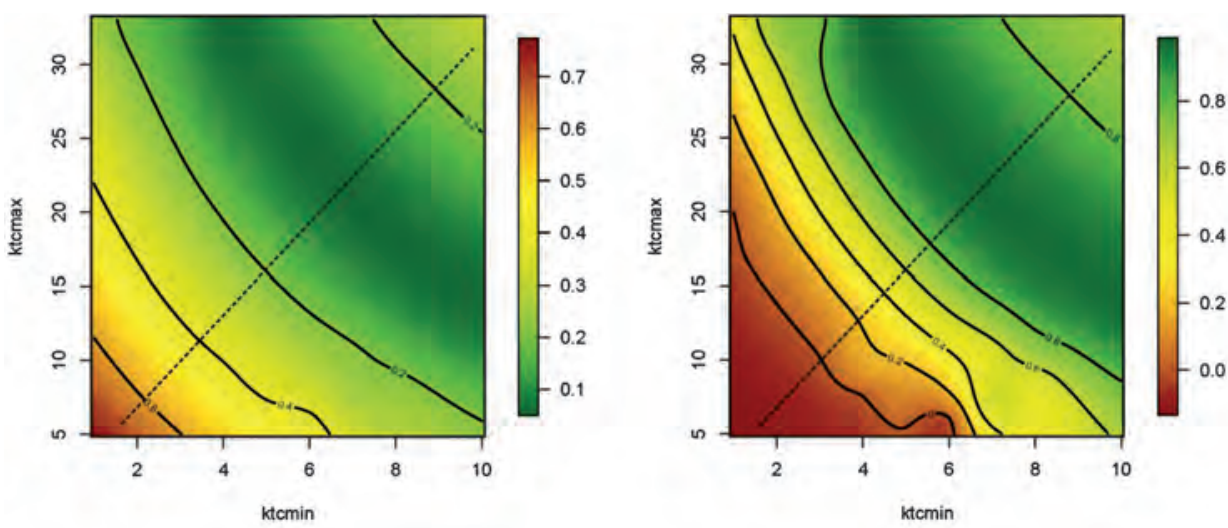

Figura 5. Calibración de los parámetros de capacidad de transporte, $k t c_{\text {max }}$ y ktc $c_{\text {min }}(m)$, en la subcuenca del río Isábena, basada en la producción de sedimentos registrada a la salida en la estación de aforo Capella (López-Tarazón et al., 2009). Ajuste del modelo para los estadísticos RRMSE (izquierda) y NS (derecha), representados en un espacio de dos dimensiones determinado por los valores de ambos parámetros. En ambos casos el color verde representa el

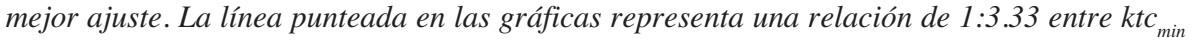
y $\mathrm{ktc}_{\text {max }}$, figura adaptada de Alatorre et al. (2010).

do a que hay un número infinito de relaciones igualmente válidas entre $\mathrm{ktc}_{\min } \mathrm{y} \mathrm{ktc}_{\max }$. Las diferencias entre estas combinaciones de parámetros se deberían restringir a la contribución relativa que tiene cada tipo de usos y coberturas de suelo a la producción de sedimentos a la salida de la cuenca. Así por ejemplo, las diferencias en la producción de sedimentos por tipos de coberturas serán mayores a medida que la relación entre los parámetros se incrementa. En otras palabras, hay que tener en cuenta que aunque la producción de sedimentos a la salida de la cuenca es correctamente predicha por el modelo, esto no significa que los patrones espaciales de la erosión y sedimentación se hayan predicho de igual manera, por lo que estos resultados deben ser tomados con mucho cuidado. En base a estas consideraciones, se ha utilizado una relación de 1:3.33 entre $\mathrm{ktc}_{\min } \mathrm{y} \mathrm{ktc}_{\max }$. Esta relación se encuentra en el rango de valores utilizados en otras áreas de estudio. Por ejemplo, Verstraeten et al. (2006) y Verstraeten (2006) utilizaron una relación de 1:3.33 en el centro del Bélgica; para áreas montañosas y áreas de suave relieve en Italia se han utilizado valores entre 1:3.80 y 1:2.20 (Van Rompaey et al., 2005); 1:3.89 para siete pequeñas cuencas en Sudáfrica (Van Rompaey et al., 2001b;Verstraeten et al., 2001); 1:2.50 en la República Checa (Van Rompaey et al., 2003a); y 1:3.33 para el suroeste de Eslovenia (Keesstra et al., 2009). Incluso hay trabajos en los que se han llegado a usar valores tan altos como 1:1.79 (Van Rompaey et al., 2001a).

Mediante la adopción de una relación fija de 1:3.33 entre $\mathrm{ktc}_{\min } \mathrm{y} \mathrm{ktc}_{\max }$ fue posible derivar un único valor para el mejor ajuste de los parámetros, correspondiente a ktc ${ }_{\min }=7 \mathrm{~m}$ $\mathrm{y}$ un $\mathrm{ktc}_{\max }=23 \mathrm{~m}$ (Fig. 6). Este conjunto de valores coinciden con los obtenidos en el proceso de calibración de una gran cuenca del Río Scheldt (Verstraeten, 2006). Los resultados de la calibración para la subcuenca del río Isábena utilizando estos valores se 

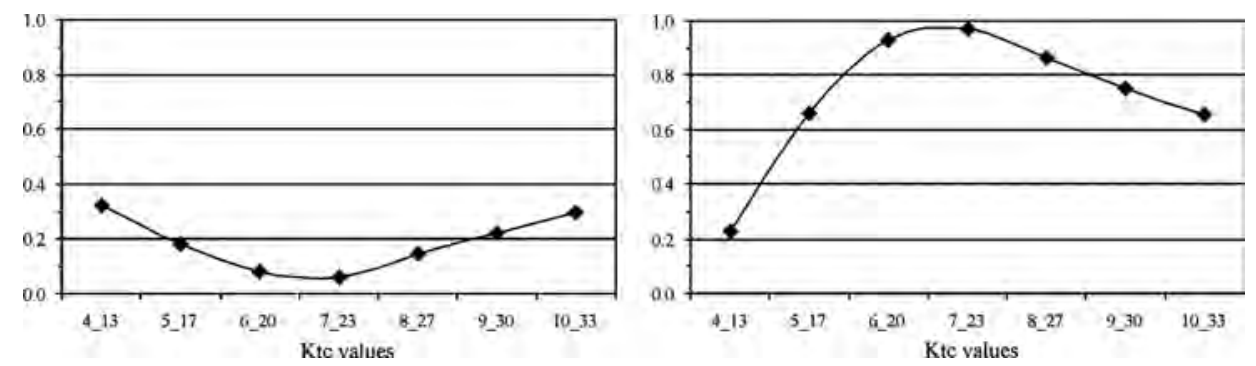

Figura 6. Calibración de los parámetros de capacidad de transporte $k t c_{\min }$ y ktc $c_{\max }(\mathrm{m})$ en la subcuenca del río Isábena basada en la producción anual de sedimentos registrada a la salida en la estación de aforo Capella (López-Tarazón et al., 2009), con una relación fija entre ambos parámetros de 1:3.33. RRMSE (izquierda) y NS (derecha), figura adaptada de Alatorre et al. (2010).

muestran en la tabla 3. Se observa que, a pesar de la variabilidad interanual que presenta la precipitación (Factor-R) en los 3 años, los valores de producción total de sedimentos (SY) y la producción específica de sedimentos (SSY) no se encuentran muy alejados de los valores observados.

Tabla 3. Producción total de sedimentos (SY) y producción específica de sedimentos (SSY) para la subcuenca del río Isábena, basada en la mejor parametrización de ktc ${ }_{\min }$ y ktc max $_{\text {a }}$ obtenida a través del proceso de calibración $\left(k t c_{\min }=7\right.$ y $\left.k t c_{\max }=23 \mathrm{~m}\right)$, tabla adaptada de Alatorre et al. (2010).

\begin{tabular}{|c|c|c|c|c|c|}
\hline Periodo & 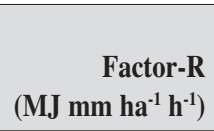 & $\begin{array}{r}\text { SY } \\
\text { predicha } \\
\left(\mathrm{Mg} \mathrm{año-1}^{-1}\right)\end{array}$ & $\begin{array}{r}\text { SY } \\
\text { observada } \\
\left(\mathrm{Mg} \mathrm{año-1}^{-1}\right)\end{array}$ & $\begin{array}{r}\text { SSY } \\
\text { predicha } \\
\left(\mathrm{Mg} \mathrm{ha}^{-1} \mathrm{año}^{-1}\right)\end{array}$ & $\begin{array}{r}\text { SSY } \\
\text { observada } \\
\left(\text { Mg ha }^{-1} a^{-1} 0^{-1}\right)\end{array}$ \\
\hline Mayo 2005 a Mayo 2006 & 937 & 108178 & 90410 & 2.47 & 2.03 \\
\hline Mayo 2006 a Mayo 2007 & 2180 & 250686 & 250290 & 5.72 & 5.62 \\
\hline Mayo 2007 a Mayo 2008 & 1900 & 219360 & 212070 & 5.01 & 4.77 \\
\hline
\end{tabular}

La calibración de los parámetros de capacidad de transporte es un aspecto fundamental de WATEM/SEDEM que todavía no ha sido resuelto. Los resultados aquí obtenidos confirman que para obtener una estimación más fiable de la distribución espacial de los procesos de erosión y sedimentación se requiere una calibración y una validación con datos espacialmente distribuidos, tal y como ya ha sido sugerido por otros autores (Takken et al. 1999, 2005; Vigiak et al., 2006).

En el caso de la cuenca experimental de Arnás, el proceso de calibración consistió en realizar un gran número de simulaciones $(n=100)$ para el periodo 1963-2003, modificando discretamente los valores de $\mathrm{ktc}_{\min } \mathrm{y} \mathrm{ktc}_{\text {max }}$. Para cada combinación de $\mathrm{ktc}_{\text {min }} \mathrm{y}$ $\mathrm{ktc}_{\max }$ se generó un mapa de redistribución neta del suelo $\left(\mathrm{Mg} \mathrm{ha}^{-1} \mathrm{año}^{-1}\right)$ con una resolución espacial de $5 \times 5 \mathrm{~m}$. Los valores de pérdida y ganancia del suelo obtenidos a partir de 

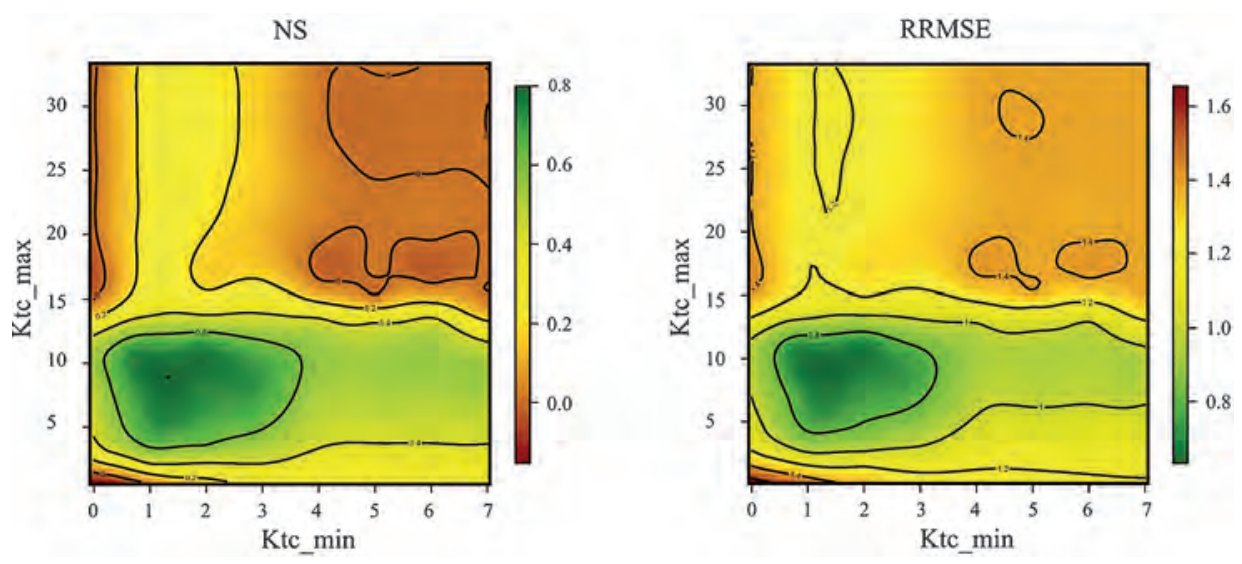

Figura 7. Calibración del parámetro de capacidad de transporte $k t c_{\min }$ y ktc $c_{\max }(\mathrm{m})$ usando tasas de redistribución de suelos derivados de los inventarios de $\mathrm{Cs}^{137}$. Superficie topográfica del error como una medida de los estadísticos NS (izquierda) y el RRMSE (derecha) en un espacio de dos dimensiones determinado por ambos parámetros. El color verde representa el mejor ajuste, figura adaptada de Alatorre et al. (2012).

los inventarios de $\mathrm{Cs}^{137}$ se compararon con cada una de las simulaciones del modelo. El grado de ajuste del modelo se calculó a partir de los estadísticos RRMSE y NS (Alatorre et al., 2012). En la Fig. 7 se observa que para ambos estadísticos existe una buena convergencia del modelo a un solo punto óptimo global que coincide con el valor máximo de NS y el mínimo de RRMSE, y que se corresponden con los valores de $\mathrm{ktc}_{\max }=9.84$ y $\mathrm{ktc}_{\min }=2.05$. La eficiencia estadística de WATEM/SEDEM para estos parámetros fue de $\mathrm{NS}=0.845$ y RRME $=0.485$, lo cual puede considerarse un muy buen resultado. Estos resultados dejan patente que el uso de datos espacialmente distribuidos de producción de sedimentos derivados de inventarios de $\mathrm{Cs}^{137}$ permite calibrar los parámetros empíricos de capacidad de transporte de WATEM/SEDEM de una forma satisfactoria, evitando el problema que se presentó en la cuenca del embalse de Barasona, al intentar calibrar los parámetros de capacidad de transporte con una sola variable (la exportación de sedimento a la salida de la cuenca). En casos como en el de la cuenca de Arnás, es posible implementar un algoritmo automatizado para encontrar el conjunto óptimo de pares de valores ktc a partir de un reducido número de procedimientos.

\subsection{Validación de WATEM/SEDEM}

La aplicación de WATEM/SEDEM a toda la cuenca del embalse de Barasona para el periodo 1955-2008 se muestra en la tabla 4. El objetivo de esta simulación fue el de obtener valores medios de SY y SSY a largo plazo, utilizando los valores medios del Factor-R para este periodo. El valor medio de SY fue de $0.56 \times 10^{-6} \mathrm{Mg}_{\text {año-1 }}$ (Tabla 4). El coeficiente de retención de sedimentos para el embalse de Barasona es de $90.15 \%$ (Almorox et al., 1994), por lo que la cantidad de sedimentos atrapados en el embalse fue de $0.50 \times 10^{-6} \mathrm{Mg}$ año ${ }^{-1}$. La SSY estimada fue de $3.73 \mathrm{Mg} \mathrm{ha}^{-1} \mathrm{año}^{-1}$, un valor muy simi- 
lar a $3.50 \mathrm{Mg} \mathrm{ha}^{-1}$ año $^{-1}$, correspondiente a la producción de sedimentos a largo plazo obtenida a partir de la batimetría del embalse de Barasona. Este resultado valida de forma significativa el proceso de calibración llevado a cabo a partir de los 3 años de registro de sedimentos a la salida de la subcuenca del río Isábena en la estación de aforo de Capella. Sin embargo, estos resultados tienen que tomarse con cuidado, ya que 3 años de datos son muy pocos para una estimación real de la producción media anual de sedimentos en una cuenca tan compleja (Alatorre et al., 2010).

Tabla 4. Predicción a largo plazo de la erosión total bruta, producción total de sedimentos $(S Y)$, producción específica de sedimentos (SSY) y relación de la entrega de sedimentos (SDR) para toda la cuenca del embalse de Barasona, basada en la mejor parametrización de ktc ${ }_{\max } y$

$k_{t c}{ }_{\text {min }}(23$ and $7 m$ respectivamente). También se indica el porcentaje de contribución de la cuenca del río Ésera, tabla adaptada de Alatorre et al. (2010).

\begin{tabular}{|c|c|c|c|c|c|c|}
\hline Periodo & 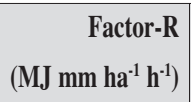 & $\begin{array}{l}\text { Erosión total bruta } \\
\qquad(\operatorname{Mg} \text { año }\end{array}$ & $\begin{array}{r}\text { SY } \\
\left(\text { Mg año }{ }^{-1}\right)\end{array}$ & $\begin{array}{r}\text { SSY } \\
\left(\mathrm{Mg} \mathrm{ha}^{-1} \mathrm{ann}^{-1}\right)\end{array}$ & $\begin{array}{r}\text { Contribución } \\
\text { Río Ésera (\%) }\end{array}$ & \\
\hline 1955-2008 & 1323 & 5907963 & 561199 & 3.73 & 70 & 10.52 \\
\hline
\end{tabular}

De igual manera, se aplicó el modelo calibrado a la cuenca experimental de Arnás y se compararon las tasas de redistribución de suelo estimados por WATEM/SEDEM con las estimaciones hechas con los inventarios de $\mathrm{Cs}^{137}$ (Fig. 8). Debe subrayarse no obstante que esta validación no se corresponde con una prueba independiente, ya que las tasas de redistribución del suelo obtenidas a partir de los inventarios de $\mathrm{Cs}^{137}$ se utilizaron en el proceso de calibración. Los resultados de la Fig. 8 muestran una fuerte relación entre las tasas de erosión estimadas por WATEM/SEDEM y las estimadas con $\mathrm{Cs}^{137}$ $\left(R^{2}=0.503\right.$, y 0.818 si se excluyen los puntos extremos), principalmente en los puntos de la ladera suroeste (matorrales) y los del fondo del valle. En general WATEM/ SEDEM realizó una ligera sobrestimación de las tasas netas de erosión. Destacan los puntos 5 y 2, localizados en la ladera con orientación noreste (bosque), que se encuentran muy alejados de la línea de ajuste. Mientras que WATEM/SEDEM predijo valores altos de erosión o sedimentación para estos puntos, se pueden seguir considerando puntos estables, tal y como reflejan las estimaciones con los inventarios de $\mathrm{Cs}^{137}$. Se intentó mejorar el grado de ajuste para estos puntos, modificando manualmente $k t c_{\text {min }}$ a valores bajos, lo cual afectó negativamente el proceso de calibración global.

De forma paralela se realizó una calibración alternativa, usando solamente los siete años de registro de sedimentos a la salida de la cuenca. En este caso se constató el mismo problema que en la calibración de la cuenca del embalse de Barasona, ya que un infinito número de combinaciones de los parámetros $\mathrm{ktc}_{\text {min }} \mathrm{y} \mathrm{ktc}_{\max }$ pueden dar resultados igualmente buenos. Esto queda reflejado en la Fig. 9, como un "valle" en el modelo estadístico RRMSE y como una "cresta" en el modelo NS. Puesto que el valor de los parámetros relativos a la capacidad de transporte está relacionado con distintos tipos de uso del suelo, seguirá siendo imposible determinar una sola combinación óptima mientras no se realice un proceso de calibración con una base de datos espacialmente distribuida. 
Figura 8. Comparación de las tasas de pérdida o ganancia del suelo estimadas por WATEM/SEDEM y las tasas de redistribución de suelos derivados de $\mathrm{Cs}^{137}$ para los valores óptimos de los parámetros. La línea continua representa un ajuste perfecto, y la línea discontinua representa la regresión lineal entre ambos datos, figura adaptada de Alatorre et al. (2012).

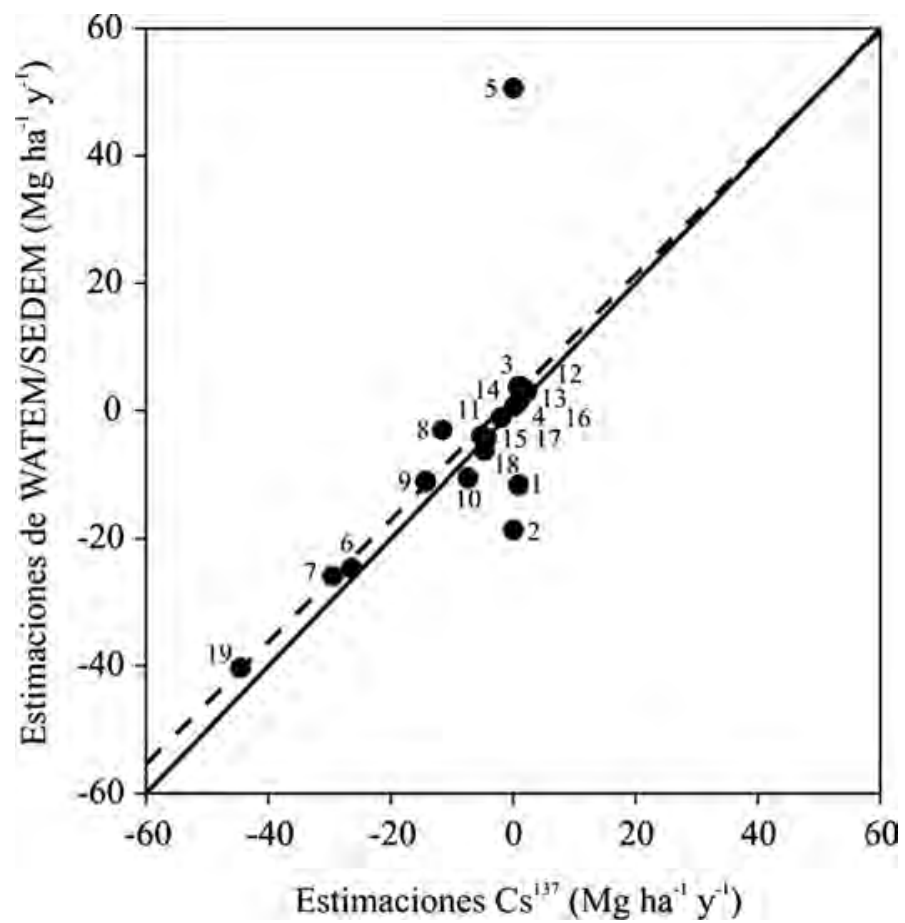

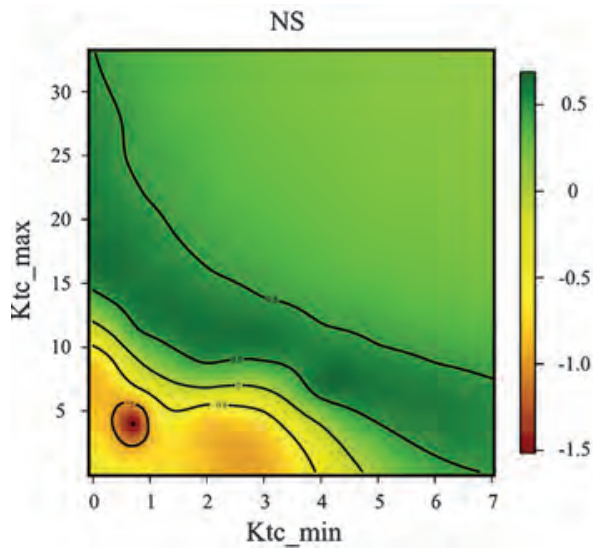

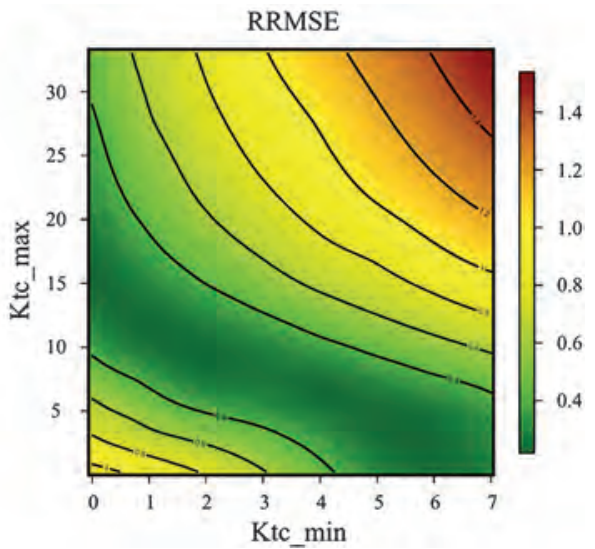

Figura 9. Calibración de los parámetros de capacidad de transporte $k t c_{\min }$ y ktc $c_{\max }(\mathrm{m})$ usando los siete años de registros de sedimentos a la salida de la cuenca de Arnás: superficie topográfica del error como una medida de los estadísticos NS (izquierda) y el RRMSE (derecha) en un espacio de dos dimensiones determinado por ambos parámetros. El color verde representa el mejor ajuste, figura adaptada de Alatorre et al. (2012). 
Finalmente, los valores de producción de sedimentos predichos por WATEM/ SEDEM con el mejor conjunto de parámetros $\mathrm{ktc}_{\min } \mathrm{y} \mathrm{ktc}_{\max }$ se validaron con los 7 años de registro de sedimentos medidos a la salida de la cuenca (Lana-Renault y Regüés, 2009). En este caso, las muestras son independientes, por lo que se puede considerar que este proceso de validación es real. Se observó una muy buena correspondencia entre los valores predichos y observados (Tabla 5 y Fig. 10), con un $\mathrm{R}^{2}=0.857(0.991$, si se excluye la peor predicción). En general el modelo estimó mejor los valores medios de producción de sedimentos, subestimó los valores por encima de la media y sobrestimó los valores por debajo de la media.

Tabla 5. Valores de precipitación acumulada $(P)$, coeficiente de escorrentía $(C E)$, coeficiente de erosividad de la lluvia (Factor-R) calculado con datos de alta frecuencia de precipitación (cada 15 min) (Angulo-Martínez y Beguería, 2009), producción de sedimentos observados (Obs. SY) y producción específica de sedimentos observados (Obs SSY) (adaptado de Lana-Renault y Regüés, 2009), producción de sedimentos simulados (Sim. SY) y producción específica de sedimentos simulados (Sim. SSY) para 7 años hidrológicos en la cuenca de Arnás. NA indica que no existen datos para un parámetro o periodo de tiempo dado, figura adaptada de Alatorre et al. (2012).

\begin{tabular}{|c|c|c|c|c|c|c|c|}
\hline $\begin{array}{l}\text { Año hidrológico } \\
\text { (Oct-Sep) }\end{array}$ & $\begin{array}{r}\mathbf{P} \\
(\mathrm{mm})\end{array}$ & $\begin{array}{r}\mathrm{CE} \\
\left(\mathrm{mm} \mathrm{mm}^{-2}\right) \\
\end{array}$ & $\begin{array}{r}\text { Factor-R } \\
\left(\mathrm{MJ} \mathrm{mm} \mathrm{ha}^{-1} \mathrm{~h}^{-1} \mathrm{ann}^{-1}\right)\end{array}$ & $\begin{array}{r}\text { Obs. SY } \\
\left(\text { Mg añ } 0^{-1}\right)\end{array}$ & $\begin{array}{r}\text { Obs. SSY } \\
\left(M_{\text {hg ha }}^{-1} \text { año }{ }^{-1}\right) \\
\end{array}$ & $\begin{array}{r}\text { Sim. SY } \\
\left(M g \text { añ } 0^{-1}\right)\end{array}$ & $\begin{array}{r}\text { Sim. SSY } \\
\left(M_{\text {ha }}^{-1}{ }^{2} \text { añ }^{-1}\right) \\
\end{array}$ \\
\hline 1999-2000 & 881 & 0.42 & 1302 & 542 & 1.91 & 473 & 1.67 \\
\hline 2000-2001 & 1353 & 0.35 & 1216 & 381 & 1.34 & 348 & 1.22 \\
\hline 2001-2002 & 765 & 0.14 & 852 & 71 & 0.25 & 244 & 0.86 \\
\hline 2002-2003 & 1043 & 0.20 & 792 & 216 & 0.76 & 227 & 0.80 \\
\hline 2003-2004 & 958 & 0.33 & 846 & 253 & 0.89 & 242 & 0.85 \\
\hline 2005-2006 & 986 & 0.25 & 715 & 116 & 0.41 & 155 & 0.55 \\
\hline 2007-2008 & 922 & 0.30 & 754 & 129 & 0.45 & 186 & 0.65 \\
\hline 1999-2008 & 986 & 0.28 & 926 & 244 & 0.86 & 268 & 0.94 \\
\hline 1963-2003 & 925 & NA & 1217 & NA & NA & 350 & 1.23 \\
\hline
\end{tabular}

La estimación para el año hidrológico 2001-2002 no es muy buena (Tabla 5) pues la producción de sedimentos observados fue particularmente baja (71 Mg año-1). Esta baja producción de sedimentos se puede atribuir a cambios en la red de drenaje causados por la acumulación de escombros después de los años 1999-2000 y 2000-2001, que fueron particularmente activos debido a la ocurrencia de tormentas intensas, tal y como queda reflejado en los elevados valores de erosividad de la lluvia (Factor-R). Los cambios morfológicos en la red de drenaje pudieron modificar temporalmente el comportamiento de los cauces, reduciendo su capacidad de transporte de sedimentos. Este proceso no puede ser capturado por el modelo, de ahí la sobrestimación de los valores predichos. La producción media de sedimentos en la cuenca experimental de Arnás para el periodo de 1999-2008 fue de $244 \mathrm{Mg}$ año-1, y la producción de sedimentos media predicha por WATEM/SEDEM fue de $268 \mathrm{Mg}$ año $^{-1}$. 
Figura 10. Relación entre el registro y la estimación de lo producción de sedimentos a la salida de la cuenca de Arnás entre los años hidrológicos 1999-2000 y 2007-2008 (de octubre a septiembre).

La línea continua representa una ajuste perfecto, y la línea discontinua la regresión lineal entre ambos valores, figura adaptada de Alatorre et al. (2012).

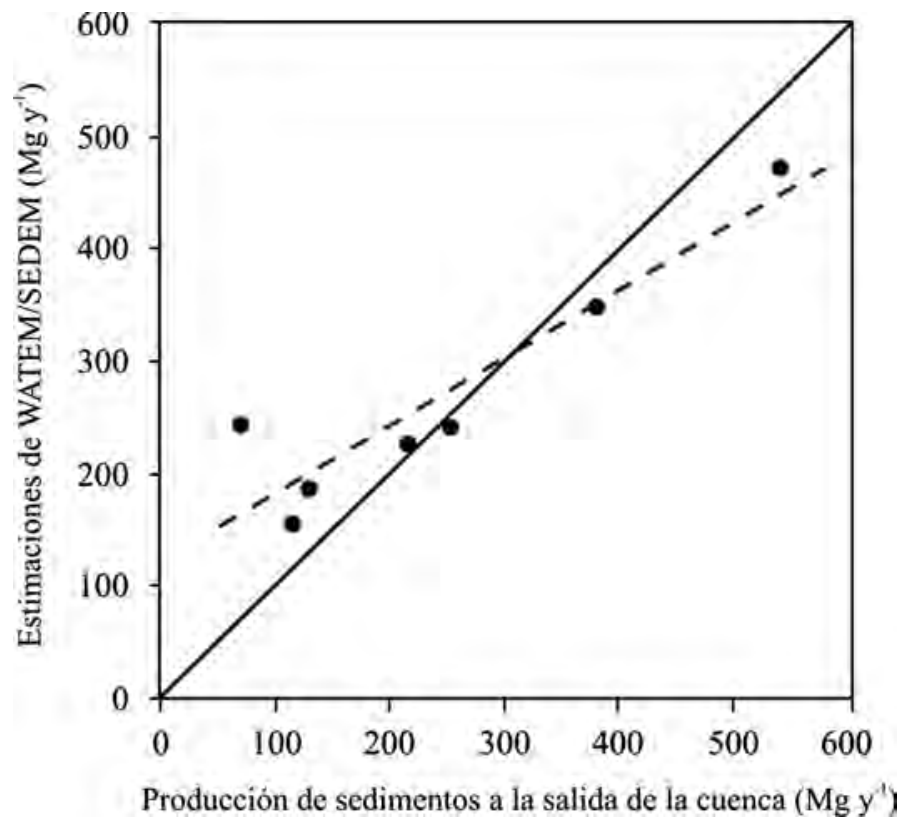

\section{Discusión y conclusiones}

En este trabajo se ha aplicado el modelo WATEM/SEDEM, un modelo empíricoconceptual espacialmente distribuido, para predecir la erosión y la producción de sedimentos en dos cuencas del Pirineo aragonés: i) la cuenca del embalse de Barasona $\left(1504 \mathrm{~km}^{2}\right)$, drenada por los ríos Ésera e Isábena, donde la base de datos para el proceso de calibración fue el registro de 3 años de sedimentos en suspensión (mayo 2005mayo 2008) a la salida de la subcuenca del Río Isábena y para la validación se utilizó el registro histórico de sedimentación del embalse de Barasona; y ii) la cuenca experimental de Arnás $\left(2.84 \mathrm{~km}^{2}\right)$, donde se contó con datos espacialmente distribuidos de tasas de erosión y sedimentación derivadas de inventarios de $\mathrm{Cs}^{137}$ para la calibración, y con el registro de seis años de sedimento en suspensión a la salida de la cuenca de Arnás para el proceso de validación.

La calibración basada en las tasas de erosión y sedimentación obtenidas a partir de los inventarios de $\mathrm{Cs}^{137}$ fue la que mejor resultados dio, ya que fue posible obtener una sola combinación óptima para los parámetros $k t c\left(k t c_{\min }=2.05 \mathrm{~m}\right.$ y $\left.k t c_{\max }=9.84 \mathrm{~m}\right)$, además de un mejor ajuste con las tasas de redistribución del suelo observadas dentro de la cuenca de Arnás. Sólo en dos sitios de la cuenca no se obtuvo una buena correspondencia entre las tasas de redistribución del suelo observadas y predichas, probablemente debido a la ocurrencia de deslizamiento sobre laderas, los cuales no son considerados por el modelo. Estos resultados pueden compararse con los obtenidos en el estudio de Feng et al. (2010), en el cual no se encontró una buena convergencia en la parametrización óptima global, ni tampoco entre las tasas de erosión predichas por WATEM/SEDEM y 
los inventarios de $\mathrm{Cs}^{137}$. Estos autores encontraron valores óptimos para $k t c_{\min } \mathrm{y} k t c_{\max } \mathrm{de}$ 6 y $7 \mathrm{~m}$ respectivamente, lo cual sugiere que establecieron poca discriminación entre los usos de suelo. El poco ajuste que se obtuvo en ese estudio se puede atribuir a que no se diseñó correctamente la toma de muestras de los inventarios de $\mathrm{Cs}^{137}$. Por ejemplo, en los usos agrícolas solo se tomaron 4 puntos, mientras que para áreas con una buena cobertura vegetal se tomaron 56 puntos. Sin lugar a dudas esto introdujo un sesgo en el proceso de calibración. Por otra parte, el algoritmo de calibración empleado no fue el óptimo, ya que se eliminó la multidimensionalidad que conlleva el tener datos espacialmente distribuidos de tasas de erosión y sedimentación, convirtiendo el problema de calibración en un problema de una sola dimensión, fijando una relación constante entre $k t c_{\min } \mathrm{y} k t c_{\max } \mathrm{y}$ haciendo caso omiso a la covarianza entre los parámetros.

Con el fin de demostrar que no se puede calibrar un modelo espacialmente distribuido únicamente con datos de producción de sedimentos a la salida del área de estudio, se realizó un proceso de calibración adicional para la cuenca de Arnás basado en la producción de sedimentos a la salida de la misma, tal y como se ha venido haciendo en muchos trabajos. Este tipo de calibración conlleva un problema fundamental asociado a la dificultad de calibrar dos parámetros relativos a la capacidad de transporte que además están relacionados con los usos de suelo. Este problema se ha venido solventando fijando una relación entre $k t c_{\min }$ y $k t c_{\text {max }}$, es decir, agregando dos parámetros en uno solo, y permitiendo así la calibración de un sólo parámetro (Verstraeten, 2006). No obstante, los resultados obtenidos en la cuenca experimental de Arnás confirman que sí es posible obtener una sola combinación óptima para los parámetros $k t c_{\min } \mathrm{y} k t c_{\max }, \mathrm{y}$ ponen de manifiesto la necesidad de trabajar con datos espacialmente distribuidos de tasa de erosión y sedimentación, como por ejemplo, inventarios de $\mathrm{Cs}^{137}$.

Los modelos espacialmente agregados proporcionan predicciones razonables de producción de sedimentos, pero no permiten identificar las principales fuentes de sedimentos. Los modelos espacialmente distribuidos, como es el caso de WATEM/SEDEM, tienen la ventaja de proporcionar información sobre las principales fuentes de sedimentos, lo cual permite una mejor aplicación de medidas de prevención y mitigación de la erosión y producción de sedimentos, así como la evaluación de los impactos que tienen los cambios de uso de suelo o de cubierta vegetal. Sin embargo, el uso de este tipo de modelos implica, por lo general, una calibración espacialmente distribuida. El uso de inventarios de $\mathrm{Cs}^{137}$ permite disponer de este tipo de información, por lo que puede considerarse una técnica muy prometedora para los procesos de calibración de modelos espacialmente distribuidos.

\section{Agradecimientos}

Este trabajo se ha llevado a cabo con el apoyo del proyecto INDICA (CGL201127753-C02-01 y -02), financiado por el Ministerio de Economía y Competitividad, el proyecto ACQWA (FP7-ENV-2007-1-212250), financiado por la Comisión Europea y un acuerdo entre el CSIC y el Ministerio de Medio Ambiente (RESEL). N. Lana-Renault ha disfrutado de un contrato de investigación en el Programa Juan de la Cierva (Minis- 
terio de Economía y Competitividad). En este artículo han participado numerosos colegas del Departamento de Procesos Geoambientales y Cambio Global del Instituto Pirenaico de Ecología (IPE), así como del Departamento de Suelo y Agua de la Estación Experimental de Aula Dei (EEAD).

\section{Referencias bibliográficas}

Alatorre, L.C., Beguería, S. 2009a. Identification of eroded areas using remote sensing in a badlands landscape on marls in the central Spanish Pyrenees. Catena 76, 182-190.

Alatorre, L.C., Beguería, S. 2009b. Identificación de zonas de erosión activa y áreas de riesgo mediante teledetección: un ejemplo en un paisaje de cárcavas sobre margas en el Pirineo Central Español. Cuadernos de Investigación Geográfica 35 (2), 171-194.

Alatorre, L.C., Beguería, S., García-Ruiz, J.M. 2010. Regional scale modeling of hillslope sediment delivery: a case study in Barasona reservoir watershed (Spain) using WATEM/SEDEM. Journal of Hydrology 391, 109-123.

Alatorre, L.C., Beguería, S., Lana-Renault, N., Navas, A., García-Ruiz, J.M. 2012. Soil erosion and sediment delivery in a mountain catchment under scenarios of land use change using a spatially distributed numerical model. Hydrology and Earth System Sciences 16, 1321-1334.

Almorox, J., De Antonio, R., Saa, A., Cruz Díaz, M., Gasco, J.M. 1994. Métodos de estimación de la erosión hídrica. Agrícola Española, Madrid, 152 pp.

Angulo-Martínez, M., Beguería, S. 2009. Estimating rainfall erosivity from daily precipitation records: a comparison between methods in the Ebro Basin (NE Spain). Journal of Hydrology $379,111-121$.

Avendaño, C., Cobo, R., Sanz, M.E., Gómez, J.L. 1997a. Capacity situation in Spanish reservoirs. Proceedings of the Nineteenth Congress on Large Dams 74 (53), 849-862.

Avendaño, C., Sanz, M.E., Cobo, R., Gómez, J.L. 1997b. Sediment yield at Spanish reservoirs and its relationships with the drainage basin area. Proceedings of the Nineteenth Congress on Large Dams 74 (54), 863-874.

Bathurst, J.C., Moretti, G., El-Hames, A., Beguería, S., García-Ruiz, J.M. 2007. Modelling the impact of forest loss on shallow landslide sediment yield, Ijuez river catchment, Spanish Pyrenees. Hydrology and Earth System Sciences 11, 569-583.

Bujan, A., Santanatoglia, O.J., Chagas, C., Massobrio, M., Castiglioni, M., Yanez, M., Ciallella, H., Fernández, J. 2003. Soil erosion evaluation in a small basin through the use of Cs-137 technique. Soil \& Tillage Research 69, 127-137.

Chueca, J., Julián, A. 2002. Rasgos climáticos del macizo de la Madaleta (Pirineo Central Español). Boletín Glaciológico Aragonés 3, 113-130.

Collins, A.L., Walling, D.E., Sichingabula, H.M., Leeks, G.J.L. 2001. Using ${ }^{137}$ Cs measurements to quantify soil erosion and redistribution rates for areas under different land use in the Upper Kaleya River basin, southern Zambia. Geoderma 104, 229-323.

Desmet, P.J.J., Govers, G. 1996. A GIS procedure for automatically calculating the USLE LS factor on topographically complex landscape units. Journal of Soil and Water Conservation 51, 427-433.

De Roo, A.P.J. 1993. Validation of the ANSWERS catchment model for runoff and soil erosion simulation in catchments in The Netherlands and the United Kingdom. IAHS Publ. 211, 465-474.

De Vente, J., Poesen, J., Verstraeten, G., Van Rompaey, A., Govers., G. 2008. Spatially distributed modelling of soil erosion and sediment yield at regional scales in Spain. Global and Planetary Change 60, 393-415. 
Feng, X., Wang, Y., Chen, L., Fu, B., Bai, G. 2010. Modeling soil erosion and its response to land-use change in hilly catchments Chinese Loess Plateau. Geomorphology 118, 239-248.

Ferro, V., Porto, P., Tusa, G. 1998. Testing a distributed approach for modeling sediment delivery. Hydrological Sciences Journal 43, 425-442.

García-Ruiz, J.M., Lasanta-Martínez, T. 1990. Land-use changes in the Spanish Pyrenees. Mountain Research and Development 10, 267-279.

García-Ruiz, J.M., Beguería, S., López Moreno, J.I., Lorente, A., Seeger, M. 2001. Los recursos hídricos superficiales del Pirineo aragonés y su evolución reciente. Geoforma, Logroño, $192 \mathrm{pp}$.

García-Ruiz, J.M., Arnáez, J., Beguería, S., Seeger, M., Martí-Bono, C., Regüés, D., LanaRenault, N., White, S. 2005. Runoff generation in an intensively disturbed, abandoned farmland catchment, Central Spanish Pyrenees. Catena 59, 79-92.

Keesstra, S.D., van Dam, O., Verstraeten, G., van Huissteden, J. 2009. Changing sediment dynamics due to natural reforestation in the Dragonja catchment, SW Slovenia. Catena 78, 60-71.

Kozak, J. 2003. Forest cover change in the Western Carpathians in the past 180 years: A case study in the Orawa region in Poland. Mountain Research and Development 23, 369-375.

Lana-Renault, N. Reguiés, D. 2007. Bedload transport under different flow condition in a humandisturbed catchment in the Central Spanish Pyrenees. Catena 7, 155-163.

Lana-Renault, N., Regüés D. 2009. Seasonal patterns of suspended sediment transport in an abandoned farmland catchment in the central Spanish Pyrenees. Earth Surface Processes and Landforms 34, 1291-1301.

Lana-Renault, N., Latron, J., Regüés, D. 2007a. Streamflow response and water-table dynamics in a sub-Mediterranean research catchment (Central Spanish Pyrenees). Journal of Hydrology 347, 497-507.

Lana-Renault, N., Regüés, D., Martí-Bono, C., Beguería, S., Latron, J., Nadal, E., Serrano, P., García-Ruiz, J.M. 2007b. Temporal variability in the relationships between precipitation, discharge and suspended sediment concentration in a small Mediterranean mountain catchment. Nordic Hydrology 38, 139-150.

Lasanta, T., Beguería, S., García-Ruiz, J.M. 2006. Geomorphic and hydrological effects of traditional shifting agriculture in a Mediterranean mountain, Central Spanish Pyrenees. Mountain Research and Development 26, 146-152.

López-Moreno, J.I., García-Ruiz, J.M. 2004. Influence of snow accumulation and snowmelt on streamflow in the Central Spanish Pyrenees. International Journal of Hydrological Sciences 49, 787-802.

López-Moreno, J.I., Beguería, S., García-Ruiz, J.M. 2002. El régimen del río Ésera, Pirineo Aragonés, y su tendencia reciente. Boletín Glaciológico Aragonés 3, 131-162.

López-Tarazón, J.A., Batalla, R.J., Vericat, D., Francke, T. 2009. Suspended sediment transport in a highly erodible catchment: the river Isábena (Central Pyrenees). Geomorphology 109, 210-221.

López-Vicente, M., Navas, A., Machín, J. 2008. Identifying erosive periods by using RUSLE factors in mountain fields of the Central Spanish Pyrenees. Hydrology and Earth System Sciences 12, 523-535.

López-Vicente, M., Lana-Renault, N., García-Ruiz, J.M., Navas, A. 2011. Assessing the potential effect of different land cover management practices on sediment yield from an abandoned farmland catchment in the Spanish Pyrenees. Journal of Soils and Sediments 11, 1440-1455.

Lorente, A., Martí-Bono, C., Beguería, S., Arnáez, J., García- Ruiz, J.M. 2000. La exportación de sedimento en suspensión en una cuenca de campos abandonados, Pirineo central español. Cuaternario y Geomorfología 14, 21-34. 
Machín, J. 1990. Mapa de suelos de Aragón (Soil map of Aragón) 1:100,000. Estación Experimental de Aula Dei, Zaragoza.

Merritt, W.S., Letcher, R.A., Jakeman, A.J. 2003. A review of erosion and sediment transport models. Environmental Modelling and Software 18, 761-799.

Nash, J.E., Sutcliffe, J.V. 1970. River flow forecasting through conceptual models: Part 1: a discussion of principles. Journal of Hydrology 10, 282-290.

Navas, A. 2002. Erosion and sedimentation features in Mediterranean landscapes assessed by fallout ${ }^{137}$ Cs. Nucleus 32, 31-37.

Navas, A., Valero, B., Machín, J., Walling, D. 1998. Los sedimentos del embalse Joaquín Costa y la historia de su depósito. Limnética 14, 93-112.

Navas, A., Soto, J., Machín, J. 2002a. Edaphic and physiographic factors affecting the distribution of natural gamma-emitting radionuclides in the soil of the Arnás catchment in the Central Spanish Pyrenees. European Journal of Soil Scences 53, 629-638.

Navas, A., Soto, J., Machín, J. 2002b. 238U, 226Ra, 210Pb, 232Th and 40K activities in soil profiles of the Flysch sector (Central Spanish Pyrenees). Applied Radiation and Isotopes 57, 579-589.

Navas, A., Machín, J., Soto, J. 2005. Assessing soil erosion in a Pyrenean mountain catchment using GIS and fallout ${ }^{137} \mathrm{Cs}$. Agriculture, Ecosystems and Environment 105, 493-506.

Navas, A., Machín, J., Beguería, S., López-Vicente, M., Gaspar, L. 2008. Soil properties and physiographic factors controlling the natural vegetation re-growth in a disturbed catchment of the Central Spanish Pyrenees. Agroforestry Systems 72, 173-185.

Renard, K.G., Foster, G.R., Weesies, G.A., Porter, J.P. 1991. RUSLE - revised universal soil loss equation. Journal of Soil and Water Conservation 46, 30-33.

Ritchie, J.C., McHenry, J.R. 1990. Application of radioactive fallout cesium-137 for measuring soil erosion and sediment accumulation rates and patterns: a review. Journal of Environmental Quality 19, 215-233.

Sanz-Montero, M., Cobo-Rayán, R., Avendaño-Salas, C., Gómez-Montaña, J. 1996. Influence of the drainage basin area on the sediment yield to Spanish reservoirs. En Proceedings of the First European Conference and Trace Exposition on Control Erosion, Sitges, Spain.

Seeger, M., Errea, M.P., Beguería, S., Arnáez, J., Martí, C., García-Ruiz, J.M. 2004. Catchment soil moisture and rainfall characteristics as determinant factors for discharge/suspended sediment hysteretic loops in a small headwater catchment in the Spanish Pyrenees. Journal of Hydrology 288, 299-311.

Taillefumier, F., Piégay, H. 2003. Contemporary land use changes in prealpine Mediterranean mountains: A multivariate GIS-based approach applied to two municipalities in the Southern French Prealps. Catena 51, 267-296.

Takken, I., Beuselinck, L., Nachtergaele, J., Govers, G., Poesen, J., Degraer, G. 1999. Spatial evaluation of a physically-based distributed erosion model (LISEM). Catena 37, 431-447.

Takken, I., Govers, G., Jetten, V., Nachtergaele, J., Steegen, A., Poesen, J. 2005. The influence of both process descriptions and runoff patterns on predictions from a spatially distributed soil erosion model. Earth Surface Processes and Landforms 30, 213-229.

Torta, G. 2004. Consequences of rural abandonment in a Northern Apennines Landscape (Tuscany, Italy). En Recent dynamics of the Mediterranean vegetation and landscape, S. Mazzoleni, G. di Pasquale, M. Mulligan, P. di Martino, F. Rego (eds.), Wiley, Chichester, pp. 157-167.

Valero-Garcés, B.L., Navas, A., Machín, J., Walling, D. 1999. Sediment sources and siltation in mountain reservoirs: a case study from the Central Spanish Pyrenees. Geomorphology 28 , 23-41.

Van Oost, K., Govers, G., Desmet, P.J.J. 2000. Evaluating the effects of landscape structure on soil erosion by water and tillage. Landscape Ecology 15, 579-591. 
Van Rompaey, A.J.J., Verstraeten, G., Van Oost, K., Govers, G., Poesen, J. 2001a. Modelling mean annual sediment yield using a distributed approach. Earth Surface Processes and Landforms 26, 1221-1236.

Van Rompaey, A., Verstraeten, G., Van Oost, K., Rozanov, A., Govers, G., Poesen, J. 2001b. Modelling sediment transport in the Jonkershoek catchment. Part 1: model calibration and validation. En Cartographic Modelling of Land Degradation, Proceedings of the Workshop held in Ghent (September 2001) in the Framework of the Bilateral Cooperation between Flanders and South-Africa, pp. 75-89.

Van Rompaey, A., Krasa, J., Dostal, T., Govers, G. 2003. Modelling sediment supply to rivers and reservoirs in Eastern Europe during and after the collectivization period. Hydrobiologia 494, 169-176.

Van Rompaey, A., Bazzoffi, P., Jones, R.J.A., Montanarella, L. 2005. Modelling sediment yields in Italian catchments. Geomorphology 65, 157-169.

Verstraeten, G. 2006. Regional scale modelling of hillslope sediment delivery with SRTM elevation data. Geomorphology 81, 128-140.

Verstraeten, G., Poesen, J. 2001. Factors controlling sediment yield from small intensively cultivated catchments in a temperate humid climate. Geomorphology 40, 123-144.

Verstraeten, G., Van Rompaey, A., Van Oost, K., Rozanov, A., Poesen, J., Govers, G. 2001. Modelling sediment transport in the Jonkershoek catchment. Part 2: evaluating the impact of possible land use changes on sediment delivery to the EersteRivier and the Jonkershoek reservoir. En Cartographic Modelling of Land Degradation, Proceedings of the Workshop held in Ghent (September 2001) in the Framework of the Bilateral Cooperation between Flanders and South-Africa, pp. 91-99.

Verstraeten, G., Van Oost, K., Van Rompaey, A., Poesen, J., Govers, G. 2002. Evaluating an integrated approach to catchment management to reduce soil loss and sediment pollution through modelling. Soil Use and Management 18, 386-394.

Verstraeten, G., Poesen, J., Gillijns, K., Govers, G. 2006. The use of riparian vegetated filter strips to reduce river sediment loads: an over-estimated control measure? Hydrological Processes 20, 4259-4267.

Verstraeten, G., Prosser, I.P., Fogarty, P. 2007. Predicting the spatial patterns of hillslope sediment delivery to river channels in the Murrumbidgee catchment, Australia. Journal of Hydrology $334,440-454$.

Vigiak, O., Sterk, G., Romanowicz, R.J., Beven, K.J. 2006. A semi-empirical model to assess uncertainty of spatial patterns of erosion. Catena 66 (3), 198-210.

Walling, D.E., Quine, T.A. 1990. Calibration of Cs-137 measurements to provide quantitative erosion rate data. Land Degradation and Rehabilitation 2, 1661-175.

Walling, D.E., Quine, T.A. 1991. Use of ${ }^{137} \mathrm{Cs}$ measurements to investigate soil erosion on arable fields in the UK: potential applications and limitations. Journal of Soil Science 42, 147-165.

Walling, D.E., He, Q. 1999. Improved models for estimating soil erosion rates from cesium-137 measurements. Journal of Environmental Quality 28, 611-622.

Walling, D.E., He, Q., Whelan, P.A. 2003. Using ${ }^{137}$ Cs measurements to validate the application of the AGNPS and ANSWERS erosion and sediment yield models in two small Devon catchments. Soil \& Tillage Research 69, 27-43. 